\title{
ANOTHER POWER IDENTITY INVOLVING BINOMIAL THEOREM AND FAULHABER'S FORMULA
}

\author{
PETRO KOLOSOV
}

Abstract. In this paper, we derive and prove, by means of Binomial theorem and Faulhaber's formula, the following identity between $m$-order polynomials in $T$

$$
\sum_{k=1}^{\ell} \sum_{j=0}^{m} A_{m, j} k^{j}(T-k)^{j}=\sum_{k=0}^{m}(-1)^{m-k} U_{m}(\ell, k) \cdot T^{k}=T^{2 m+1}, \ell=T \in \mathbb{N} .
$$

\section{Contents}

1. Introduction and Main results 1

$\begin{array}{lll}\text { 1.1. } & \text { Error of approximation } & 12\end{array}$

2. Acknowledgements 19

$\begin{array}{lll}3 . & \text { Conclusion } & 19\end{array}$

\begin{tabular}{lll}
\hline 4. Supplementary Files & 19
\end{tabular}

\begin{tabular}{ll}
\hline References & 19
\end{tabular}

\section{Introduction AND MAIN RESUlts}

The following identity holds in $m$-order polynomials $P_{m}(\ell, T)$

$$
P_{m}(\ell, T):=\sum_{k=1}^{\ell} \sum_{j=0}^{m} A_{m, j} k^{j}(T-k)^{j}=\left.\sum_{k=0}^{m}(-1)^{m-k} U_{m}(\ell, k) \cdot T^{k}\right|_{\ell=T \in \mathbb{N}}=T^{2 m+1},
$$

where $\ell \in \mathbb{N}_{1}, m \in \mathbb{N}_{1}$ and $T \in \mathbb{R}$ and $U_{m}(\ell, k), A_{m, j}$ are coefficients dependent on $\ell$ and $m$, respectively. The term $(-1)^{m-k}$ appears in r.h.s of the expression 1.1 by means of sign-changing inside $\sum_{j=0}^{m} A_{m, j} k^{j}(T-k)^{j}$ by Binomial expansion of $(T-k)^{j}$.

We start our discussion concerning the identity (1.1) from the derivation of the partial case of the polynomial $P_{m}(\ell, T)$ for $m=1$, by means of Faulhaber's identity for $n^{3}$. The terms of the of the polynomial $P_{1}(T, T)=\sum_{k=1}^{T} \sum_{j=0}^{1} A_{1, j} k^{j}(T-k)^{j}=T^{3}$ over $k$ produce the $T$-th row of the sequence A287326, starting from $k=1$, [3]. To derive the partial case of the polynomial $P_{m}(\ell, T)$ for $m=1$, let's consider the Faulhaber's identities [1] for odd powers $n^{2 m+1}, m \in \mathbb{N}_{0}$

$$
\begin{cases}n^{1} & =\left(\begin{array}{c}
n \\
1
\end{array}\right) \\
n^{3} & =6\left(\begin{array}{c}
n+1 \\
3
\end{array}\right)+\left(\begin{array}{c}
n \\
1
\end{array}\right) \\
n^{5} & =120\left(\begin{array}{c}
n+2 \\
5
\end{array}\right)+30\left(\begin{array}{c}
n+1 \\
3
\end{array}\right)+\left(\begin{array}{c}
n \\
1
\end{array}\right) \\
& \vdots \\
n^{2 m-1} & =\sum_{1 \leq k \leq m}(2 k-1) ! T(2 m, 2 k)\left(\begin{array}{c}
n+k-1 \\
2 k-1
\end{array}\right)\end{cases}
$$

2010 Mathematics Subject Classification. $11 \mathrm{C} 08$ (primary), 41A10 (secondary).

Key words and phrases. Faulhaber's formula, Faulhaber's theorem, Binomial Theorem, Binomial coefficient, Binomial distribution, Binomial identities, Power Sums, Finite differences. 
The coefficients $T(m, k)$ in these formula are related to what Riordan [19] has called central factorial numbers of the second kind. In his notation,

$$
x^{m}=\sum_{1 \leq k \leq m} T(m, k) x^{[k]}, x^{[k]}=x\left(x+\frac{k}{2}-1\right)\left(x+\frac{k}{2}-2\right) \cdots\left(x+\frac{k}{2}+1\right)
$$

The coefficients $T(2 m, 2 k)$ are always integers, because the $x^{[k+2]}=x^{[k]}\left(x^{2} / k^{4}\right)$ implies recurrence

$$
T(2 m+2,2 k)=k^{2} T(2 m, 2 k)+T(2 m, 2 k-2) .
$$

Central factorial numbers of the second kind can be calculated as follows:

$$
(2 k-1) ! T(2 n, 2 k)=\frac{1}{r} \sum_{j=0}^{r}(-1)^{j}\left(\begin{array}{c}
2 r \\
j
\end{array}\right)(r-j)^{2 n},
$$

where $r=n-k+1$. The formula (1.3) was derived by Peter Luschny in [20].

The forward finite difference of odd power could be reached introducing the $r \in \mathbb{N}_{1}$ to the lower index of binomial coefficient of $(1.2)$ the following way

$$
\begin{cases}r=1: \Delta n^{1}=\left(\begin{array}{c}
n \\
1-r
\end{array}\right) & =\left(\begin{array}{c}
n \\
0
\end{array}\right) \\
r=1: \Delta n^{3}=6\left(\begin{array}{c}
n+1 \\
3-r
\end{array}\right)+\left(\begin{array}{c}
n \\
1-r
\end{array}\right) & =6\left(\begin{array}{c}
n+1 \\
2
\end{array}\right)+\left(\begin{array}{c}
n \\
0
\end{array}\right) \\
r=1: \Delta n^{5}=120\left(\begin{array}{c}
n+2 \\
5-r
\end{array}\right)+30\left(\begin{array}{c}
n+1 \\
3-r
\end{array}\right)+\left(\begin{array}{c}
n \\
1-r
\end{array}\right) & =120\left(\begin{array}{c}
n+2 \\
4
\end{array}\right)+30\left(\begin{array}{c}
n+1 \\
2
\end{array}\right)+\left(\begin{array}{c}
n \\
0
\end{array}\right) \\
r=1: \Delta n^{2 m-1}=\sum_{1 \leq k \leq m}(2 k-1) ! T(2 m, 2 k)\left(\begin{array}{c}
n+k-1 \\
2 k-1-r
\end{array}\right) & =\sum_{1 \leq k \leq m}(2 k-1) ! T(2 m, 2 k)\left(\begin{array}{c}
n+k-1 \\
2 k-1-1
\end{array}\right)\end{cases}
$$

By the dentity $\Delta f(x)=\nabla f(x+1), h=1$, backward differences could be reached as well,

$$
\begin{cases}r=1: \nabla(n+1)^{1}=\left(\begin{array}{c}
n \\
1-r
\end{array}\right) & =\left(\begin{array}{c}
n \\
0
\end{array}\right) \\
r=1: \nabla(n+1)^{3}=6\left(\begin{array}{c}
n+1 \\
3-r
\end{array}\right)+\left(\begin{array}{c}
n \\
1-r
\end{array}\right) & =6\left(\begin{array}{c}
n+1 \\
2
\end{array}\right)+\left(\begin{array}{c}
n \\
0
\end{array}\right) \\
r=1: \nabla(n+1)^{5}=120\left(\begin{array}{c}
n+2 \\
5-r
\end{array}\right)+30\left(\begin{array}{c}
n+1 \\
3-r
\end{array}\right)+\left(\begin{array}{c}
n \\
1-r
\end{array}\right) & =120\left(\begin{array}{c}
n+2 \\
4
\end{array}\right)+30\left(\begin{array}{c}
n+1 \\
2
\end{array}\right)+\left(\begin{array}{c}
n \\
0
\end{array}\right) \\
r=1: \nabla(n+1)^{2 m-1}=\sum_{1 \leq k \leq m}(2 k-1) ! T(2 m, 2 k)\left(\begin{array}{c}
n+k-1 \\
2 k-1-r
\end{array}\right) & =\sum_{1 \leq k \leq m}(2 k-1) ! T(2 m, 2 k)\left(\begin{array}{c}
n+k-1 \\
2 k-1-1
\end{array}\right)\end{cases}
$$

Continuing similarly, for every $r \geq 1$ we get $\Delta^{r} n^{2 m-1}$ and $\nabla^{r} n^{2 m-1}$. To derive the polynomial $P_{m}(T, T)=T^{2 m+1}, T \in \mathbb{N}_{1}$, for $m=1$, recall the Faulhaber's identity $\Delta n^{3}=\nabla(n+1)^{3}=$ $6\left(\begin{array}{c}n+1 \\ 2\end{array}\right)+\left(\begin{array}{l}n \\ 0\end{array}\right)$, thus, the perfect cube in $T$ is:

$$
\begin{aligned}
T^{3} & =\sum_{k=0}^{T-1} \Delta T^{3}(k)=\sum_{k=0}^{T-1} 6\left(\begin{array}{c}
k+1 \\
2
\end{array}\right)+\left(\begin{array}{l}
k \\
0
\end{array}\right) \\
& =\sum_{k=1}^{T} \nabla(T+1)^{3}(k)=\sum_{k=1}^{T} 6\left(\begin{array}{c}
k+1 \\
2
\end{array}\right)+\left(\begin{array}{l}
k \\
0
\end{array}\right) .
\end{aligned}
$$

Let's rewrite the expression 1.4 , taking to the attention the following identity $\left(\begin{array}{c}k+2 \\ 2\end{array}\right)=1+2+$ $\cdots+(k+2)$, thus

$$
\begin{aligned}
T^{3} & =(1+6 \cdot 0)+(1+6 \cdot 0+6 \cdot 1)+\cdots+(1+6 \cdot 0+\cdots+6 \cdot(T-1)) \\
& =(1+6 \cdot 1)+(1+6 \cdot 1+6 \cdot 2)+\cdots+(1+6 \cdot 1+\cdots+6 \cdot T)
\end{aligned}
$$

Factorising the expression 1.5 we get

$$
\begin{aligned}
T^{3} & =T+(T-0) \cdot 6 \cdot 0+(T-1) \cdot 6 \cdot 1+\cdots+(T-(T-1)) \cdot 6 \cdot(T-1) \\
& =T+(T-1) \cdot 6 \cdot 1+(T-2) \cdot 6 \cdot 2+\cdots+(T-T) \cdot 6 \cdot T
\end{aligned}
$$


Let's apply a compact sigma notation on the expression $(1.6)$, we have

$$
\begin{aligned}
T^{3} & =T+\sum_{k=0}^{T-1} 6 k(T-k)=\sum_{k=0}^{T-1} 6 k(T-k)+1 \\
& =T+\sum_{k=1}^{T} 6 k(T-k)=\sum_{k=1}^{T} 6 k(T-k)+1=P_{1}(T, T),
\end{aligned}
$$

see (1.1) for $P_{1}(T, T)$.

Although, the expression (1.7) is derived, essentially, from identity in finite differences of cubes, such as $T^{3}=\sum_{k=0}^{T-1} \Delta T^{3}(k)$ and $T^{3}=\sum_{k=1}^{T} \nabla(T+1)^{3}(k)$, the finite differences $\Delta T^{3}(k) \neq 6 k(T-$ $k)+1$ and $\nabla(T+1)^{3}(k) \neq 6 k(T-k)+1$. Therefore, we have reached the generating function of A287326 in r.h.s of 1.7). The corresponding coefficients $A_{m, j}$ in the definition $\sum_{j=0}^{1} A_{1, j} k^{j}(T-$ $k)^{j}=1+6 k(T-k)$ of generating function are: $A_{1,0}=1, A_{1,1}=6$. Note that by definition the polynomials $\sum_{j=0}^{m} A_{m, j} k^{j}(T-k)^{j}$ should be displayed starting from the $A_{m, 0} k^{0}(T-k)^{0}$, we will denote these polynomials starting from the $m$-th term, as the order of summation doesn't change it's result. Let's construct the triangle A287326, every $(\ell=T)$-th row sum starting from $k=1$ gives the terms of polynomial $P_{1}(\ell, T)=\sum_{k=1}^{\ell} \sum_{j=0}^{1} A_{1, j} k^{j}(T-k)^{j}=\sum_{k=1}^{\ell} 6 k(T-k)+1$, starting from $k=1$. We review the triangle A287326 in context of the partial case of polynomial $P_{m}(\ell, T), m=1, \ell=T \in \mathbb{N}_{0}$

$$
\begin{aligned}
& \ell=T=0 \\
& 1 \\
& \ell=T=1 \\
& 1 \quad 1 \\
& \ell=T=2 \\
& 1 \quad 7 \\
& \ell=T=3 \\
& 1 \\
& \ell=T=4 \\
& \ell=T=5 \quad 1
\end{aligned}
$$

19

13

25

13

$37 \quad 25 \quad 1$

Table 1. Triangle generated by the polynomial $\sum_{j=0}^{1} A_{1, j} k^{j}(T-k)^{j}=6 k(T-k)+1,0 \leq$ $k \leq \ell=T \in \mathbb{N}_{0}$, sequence A287326 in OEIS. Summation of the $(\ell=T)$-th row terms from $k=1$ gives $P_{1}(T, T)=T^{3}$, see 11.1 .

The sum of the $(\ell=T)$-th row terms of table 1 starting from $k=1$ generates the partial case of (1.1) for $m=1$ and $\ell=T \in \mathbb{N}$, that is $P_{1}(T, T)=T^{3}$. Binomial distribution of the row terms of table 1 can be easily proven by reviewing of its generating function $\sum_{j=0}^{1} A_{1, j} k^{j}(T-k)^{j}=$ $6 k(T-k)+1=6 k T-6 k^{2}+1$, which is parabolic for every given variable $T$, and, therefore, is symmetrical over $\frac{T}{2}$. Hence, the identity follows

$$
D_{1}(T, k):=\sum_{j=0}^{1} A_{1, j} k^{j}(T-k)^{j}, D_{1}(T, k)=D_{1}(T-k, k)
$$


Below we show initial ten polynomials, generated by the partial case of 1.1 for $m=1$ in $\ell=$ $1,2, \ldots, 10$ :

$$
\begin{aligned}
P_{1}(\ell, T)= & \sum_{k=1}^{\ell} \sum_{j=0}^{1} A_{1, j} k^{j}(T-k)^{j}=\sum_{k=1}^{\ell} 6 k(T-k)+1=\sum_{k=0}^{1}(-1)^{1-k} U_{1}(\ell, k) \cdot T^{k} \\
& \begin{cases}\ell=1:-5+6 T & =-U_{1}(1,0) \cdot T^{0}+U_{1}(1,1) \cdot T^{1}=P_{1}(1, T) \\
\ell=2:-28+18 T & =-U_{1}(2,0) \cdot T^{0}+U_{1}(2,1) \cdot T^{1}=P_{1}(2, T) \\
\ell=3:-81+36 T & =-U_{1}(3,0) \cdot T^{0}+U_{1}(3,1) \cdot T^{1}=P_{1}(3, T) \\
\ell=4:-176+60 T & =-U_{1}(4,0) \cdot T^{0}+U_{1}(4,1) \cdot T^{1}=P_{1}(4, T) \\
\ell=5:-325+90 T & =-U_{1}(5,0) \cdot T^{0}+U_{1}(5,1) \cdot T^{1}=P_{1}(5, T) \\
\ell=6:-540+126 T & =-U_{1}(6,0) \cdot T^{0}+U_{1}(6,1) \cdot T^{1}=P_{1}(6, T) \\
\ell=7:-833+168 T & =-U_{1}(7,0) \cdot T^{0}+U_{1}(7,1) \cdot T^{1}=P_{1}(7, T) \\
\ell=8:-1216+216 T & =-U_{1}(8,0) \cdot T^{0}+U_{1}(8,1) \cdot T^{1}=P_{1}(8, T) \\
\ell=9:-1701+270 T & =-U_{1}(9,0) \cdot T^{0}+U_{1}(9,1) \cdot T^{1}=P_{1}(9, T) \\
\ell=10:-2300+330 T & =-U_{1}(10,0) \cdot T^{0}+U_{1}(10,1) \cdot T^{1}=P_{1}(10, T)\end{cases} \\
= & T^{3} \text { as } T \rightarrow \ell .
\end{aligned}
$$

The coefficients $U_{1}(\ell, k), 0 \leq k \leq 1$ in $(1.9)$ are terms of the sequence A320047. One would ask, "Why to show the identity (1.1) in terms of $(\ell=T)$-th row of table 1 we used the condition $\ell=T \in \mathbb{N}_{0}$, but to show the identity (1.1) in terms of $(1.9)$ we apply the condition $T \rightarrow \ell$, and $\ell \in \mathbb{N}$ ?" - We used the condition $T \rightarrow \ell$, and $\ell \in \mathbb{N}$ to show the identity (1.1) in terms of (1.9) as, by definition $T \in \mathbb{R}$ in $(1.1)$ and the variable $\ell \in \mathbb{N}$, since the coefficient $U_{m}(\ell, k)$ is dependent on $\ell$, and, therefore, $\ell$ is always $\mathbb{N}_{1}$, so if we speak about approximation of monomial $T^{2 m+1}$ by polynomial $\sum_{k=0}^{1}(-1)^{1-k} U_{1}(\ell, k) \cdot T^{k}$ in some particular point $\ell \in \mathbb{N}$, we say that $T \rightarrow \ell$. The binomials, listed in $(1.9)$ are linear approximations of monomial $T^{3}$ in some neighborhood of the point $\ell \in \mathbb{N}$ and $T \rightarrow \ell$. The following figure graphically shows the binomials $\sum_{k=0}^{1}(-1)^{1-k} U_{1}(\ell, k) \cdot T^{k}, \ell=1,2,3$ among the cubic curve in $T$

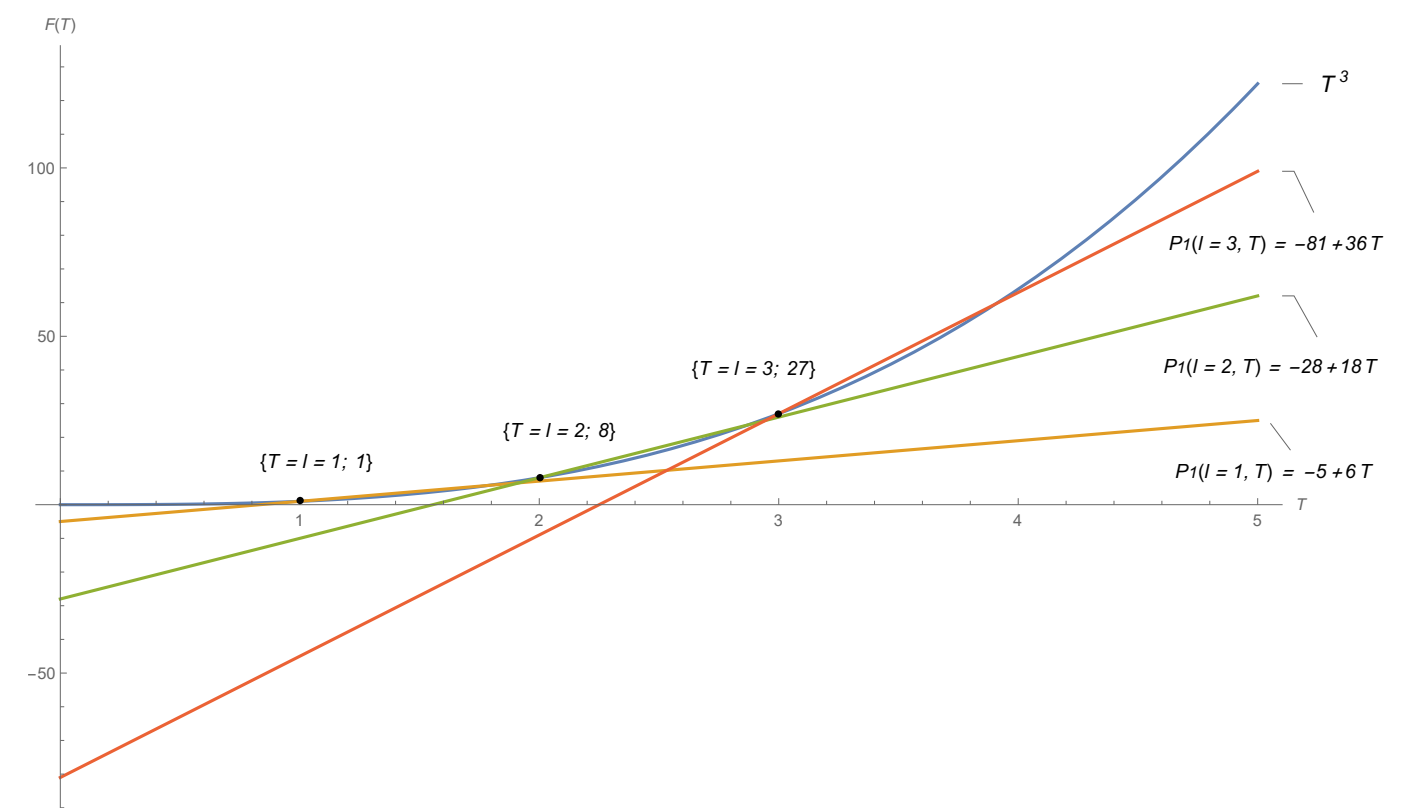

Figure 1. Linear approximations of the monomial $T^{3}$ by binomials $P_{1}(1, T), P_{1}(2, T), P_{1}(3, T)$ in some neighborhood of the point $\ell=1,2,3$ with $T \rightarrow \ell$, see (1.11. 
Now, we have derived and discussed the pattern A287326, generated by the $\sum_{j=0}^{1} A_{1, j} k^{j}(T-$ $k)^{j}=6 k(T-k)+1,0 \leq k \leq \ell=T \in \mathbb{N}_{0}$. Let's find analogs of the pattern A287326 for $m>1$. To find such analogs, let's determine the coefficients $A_{2,0}, A_{2,1}, A_{2,2}$ in the polynomial $\sum_{j=0}^{2} A_{2, j} k^{j}(T-k)^{j}$, such that $P_{2}(\ell, T)=\sum_{k=1}^{\ell} \sum_{j=0}^{2} A_{2, j} k^{j}(T-k)^{j}=T^{5}$, as $\ell=T \in \mathbb{N}$, by the (1.2). Firstly, to determine the coefficients coefficients $A_{2,0}, A_{2,1}, A_{2,2}$, let's rewrite the polynomial $P_{2}(\ell, T)=\sum_{k=1}^{\ell} \sum_{j=0}^{2} A_{2, j} k^{j}(T-k)^{j}$, in extended view as follows

$$
\begin{aligned}
& \sum_{k=1}^{\ell} \sum_{j=0}^{2} A_{2, j} k^{j}(T-k)^{j} \\
= & A_{2,2} \sum_{1 \leq k \leq \ell} k^{2}(T-k)^{2}+A_{2,1} \sum_{1 \leq k \leq \ell} k^{1}(T-k)^{1}+A_{2,0} \sum_{1 \leq k \leq \ell} k^{0}(T-k)^{0} \\
= & A_{2,2} \sum_{1 \leq k \leq \ell} k^{2}\left(T^{2}-2 T k+k^{2}\right)+A_{2,1} \sum_{1 \leq k \leq \ell} k T-k^{2}+A_{2,0} \sum_{1 \leq k \leq \ell} k^{0}(T-k)^{0} \\
= & A_{2,2} \sum_{1 \leq k \leq \ell} k^{2} T^{2}-2 T k^{3}+k^{4}+A_{2,1} \sum_{1 \leq k \leq \ell} k T-k^{2}+A_{2,0} \sum_{1 \leq k \leq \ell} 1 \\
= & A_{2,2} T^{2}\left(\sum_{1 \leq k \leq \ell} k^{2}\right)-2 A_{2,2} T\left(\sum_{1 \leq k \leq \ell} k^{3}\right)+A_{2,2}\left(\sum_{1 \leq k \leq \ell} k^{4}\right)+A_{2,1} T\left(\sum_{1 \leq k \leq \ell} k\right) \\
& -A_{2,1}\left(\sum_{1 \leq k \leq \ell} k^{2}\right)+A_{2,0}\left(\sum_{1 \leq k \leq \ell}\right)=T^{5}, \text { as } \ell=T \in \mathbb{N} .
\end{aligned}
$$

Above derivation could be generalised in terms of Binomial coefficients and Faulhaber's sum for every $m \in \mathbb{N}_{0}$ as follows

$$
\sum_{s=0}^{m} \sum_{j=0}^{m-s}(-1)^{j} A_{m, m-s}\left(\begin{array}{c}
m-s \\
j
\end{array}\right)\left(\sum_{k=1}^{\ell} k^{m+s}\right) T^{m+s}
$$

Note that the part $A_{2,0} \sum_{1 \leq k \leq \ell} k^{0}(T-k)^{0}$ of 1.10 gives an indeterminate form as $k=T$ since the $A_{2,0}(T-T)^{0} k^{0}$ contains the term $(T-T)^{0}=0^{0}$. Some textbooks leave the quantity $0^{0}$ undefined, because the functions $x^{0}$ and $0^{x}$ have different limiting values when $x$ decreases to 0 . For our purposes we will use the convention:

$$
\forall x: x^{0}=1,
$$

as it is a common agreement, see [16. Above we have derived an expression containing sums of powers of successive natural exponents, where the powers are $\{1,2,3,4\}$. These formulae contain so-called Bernoulli numbers, [7]. By Faulhaber's formula, the sums of successive powers in $k$ are following

$$
\begin{aligned}
& \sum_{1 \leq k \leq \ell} k=\frac{\ell^{2}+\ell}{2}, \\
& \sum_{1 \leq k \leq \ell} k^{2}=\frac{2 \ell^{3}+3 \ell^{2}+\ell}{6}, \\
& \sum_{1 \leq k \leq \ell} k^{3}=\frac{\ell^{4}+2 \ell^{3}+\ell^{2}}{4}, \\
& \sum_{1 \leq k \leq \ell} k^{4}=\frac{6 \ell^{5}+15 \ell^{4}+10 \ell^{3}-\ell}{30} .
\end{aligned}
$$


Next, we substitute the identities (1.11), into 1.10, respectively

$$
\begin{aligned}
A_{2,2} T^{2}\left(\frac{2 \ell^{3}+3 \ell^{2}+\ell}{6}\right) & -2 A_{2,2} T\left(\frac{\ell^{4}+2 \ell^{3}+\ell^{2}}{4}\right)+A_{2,2}\left(\frac{6 \ell^{5}+15 \ell^{4}+10 \ell^{3}-\ell}{30}\right) \\
& +A_{2,1} T\left(\frac{\ell^{2}+\ell}{2}\right)-A_{2,1}\left(\frac{2 \ell^{3}+3 \ell^{2}+\ell}{6}\right)+A_{2,0} \ell .
\end{aligned}
$$

Factorising the expression 1.12 and collecting the terms under common divisor with set $\ell=T \in \mathbb{N}$, we get

$$
\frac{A_{2,2} T^{5}-A_{2,2} T+30 A_{2,0}}{30}+A_{2,1} \frac{T^{3}-T}{6}=T^{5}
$$

In order to satisfy 1.13 for each $\ell=T \in \mathbb{N}$, coefficients $A_{2,0}, A_{2,1}, A_{2,2}$ should be a solutions of following system of equations

$$
\begin{cases}\frac{1}{30} A_{2,2} & =1 \\ A_{2,1} & =1 \\ 30 A_{2,0}-A_{2,2} & =0 .\end{cases}
$$

The solutions to the system (1.14) are following: $A_{2,2}=30, A_{2,1}=0, A_{2,0}=1$. Hereby, polynomial $\sum_{j=0}^{2} A_{2, j} k^{j}(T-k)^{j}$ takes the form

$$
\sum_{j=0}^{2} A_{2, j} k^{j}(T-k)^{j}=30 k^{2}(T-k)^{2}+1 .
$$

\begin{tabular}{|c|c|c|c|c|c|c|c|c|}
\hline$\ell=T=0$ & & & & 1 & & & & \\
\hline$\ell=T=1$ & & & 1 & & 1 & & & \\
\hline$\ell=T=2$ & & 1 & & 31 & & 1 & & \\
\hline$\ell=T=3$ & 1 & & 121 & & 121 & & 1 & \\
\hline$\ell=T=4$ & 1 & 271 & & 481 & & 271 & & 1 \\
\hline$\ell=T=5 \quad 1$ & 481 & & 1081 & & 1081 & & 481 & 1 \\
\hline
\end{tabular}

Let's construct the triangle, every $(\ell=T)$-th row sum starting from $k=1$ gives the terms of polynomial $P_{2}(\ell, T)=\sum_{k=1}^{\ell} \sum_{j=0}^{2} A_{2, j} k^{j}(T-k)^{j}=\sum_{k=1}^{\ell} 30 k^{2}(T-k)^{2}+1$, over $k$ in range $1 \leq k \leq \ell=T \in \mathbb{N}_{0}$. We review the triangle in context of the partial case of polynomial $P_{m}(\ell, T), m=2, \ell=T \in \mathbb{N}_{0}$

Table 2. Triangle generated by the polynomial $\sum_{j=0}^{2} A_{2, j} k^{j}(T-k)^{j}=30 k^{2}(T-k)^{2}+1,0 \leq$ $k \leq \ell=T \in \mathbb{N}_{0}$, sequence A300656 in OEIS, 8 8 . Summation of the $(\ell=T)$-th row terms from $k=1$ gives $P_{2}(T, T)=T^{5}$, see 1.1 .

By the identity (1.1) in polynomials $P_{m}(\ell, T)$, the sum of the $(T=\ell)$-th row terms of the table 2 over $k$ from 1 to $\ell=T \in \mathbb{N}$ gives $P_{2}(T, T)=T^{5}$. Below we show initial ten polynomials, generated 
by the partial case of 1.1$)$ for $m=2$ in $\ell=1,2, \ldots, 10$ :

$$
\begin{aligned}
& P_{2}(\ell, T)=\sum_{k=1}^{\ell} \sum_{j=0}^{2} A_{2, j} k^{j}(T-k)^{j}=\sum_{k=1}^{\ell} 30 k^{2}(T-k)^{2}+1=\sum_{k=0}^{2}(-1)^{2-k} U_{2}(\ell, k) \cdot T^{k} \\
& \qquad \begin{array}{ll}
\ell=1: 31-60 T+30 T^{2} & =U_{2}(1,0) \cdot T^{0}-U_{2}(1,1) \cdot T^{1}+U_{2}(1,2) \cdot T^{2}=P_{2}(1, T) \\
\ell=2: 512-540 T+150 T^{2} & =U_{2}(2,0) \cdot T^{0}-U_{2}(2,1) \cdot T^{1}+U_{2}(2,2) \cdot T^{2}=P_{2}(2, T) \\
\ell=3: 2943-2160 T+420 T^{2} & =U_{2}(3,0) \cdot T^{0}-U_{2}(3,1) \cdot T^{1}+U_{2}(3,2) \cdot T^{2}=P_{2}(3, T) \\
\ell=4: 10624-6000 T+900 T^{2} & =U_{2}(4,0) \cdot T^{0}-U_{2}(4,1) \cdot T^{1}+U_{2}(4,2) \cdot T^{2}=P_{2}(4, T) \\
\ell=5: 29375-13500 T+1650 T^{2} & =U_{2}(5,0) \cdot T^{0}-U_{2}(5,1) \cdot T^{1}+U_{2}(5,2) \cdot T^{2}=P_{2}(5, T) \\
\ell=6: 68256-26460 T+2730 T^{2} & =U_{2}(6,0) \cdot T^{0}-U_{2}(6,1) \cdot T^{1}+U_{2}(6,2) \cdot T^{2}=P_{2}(6, T) \\
\ell=7: 140287-47040 T+4200 T^{2} & =U_{2}(7,0) \cdot T^{0}-U_{2}(7,1) \cdot T^{1}+U_{2}(7,2) \cdot T^{2}=P_{2}(7, T) \\
\ell=8: 263168-77760 T+6120 T^{2} & =U_{2}(8,0) \cdot T^{0}-U_{2}(8,1) \cdot T^{1}+U_{2}(8,2) \cdot T^{2}=P_{2}(8, T) \\
\ell=9: 459999-121500 T+8550 T^{2} & =U_{2}(9,0) \cdot T^{0}-U_{2}(9,1) \cdot T^{1}+U_{2}(9,2) \cdot T^{2}=P_{2}(9, T) \\
\ell=10: 760000-181500 T+11550 T^{2} & =U_{2}(10,0) \cdot T^{0}-U_{2}(10,1) \cdot T^{1}+U_{2}(10,2) \cdot T^{2}=P_{2}(10, T)
\end{array} \\
& =T^{5} \text { as } T \rightarrow \ell .
\end{aligned}
$$

The coefficients $U_{2}(\ell, k), 0 \leq k \leq 2$ in (1.16) are terms of the sequence A316349, 17]. Above polynomials are approximations of monomial $T^{5}$ in some neighborhood of the point $T \rightarrow \ell$, the following graph shows corresponding polynomials $\sum_{k=1}^{\ell} \sum_{j=0}^{2} A_{2, j} k^{j}(T-k)^{j}=\sum_{k=0}^{2}(-1)^{2-k} U_{2}(\ell, k) \cdot T^{k}, \ell=$ $1,2, .$. and the $T^{5}$ curve

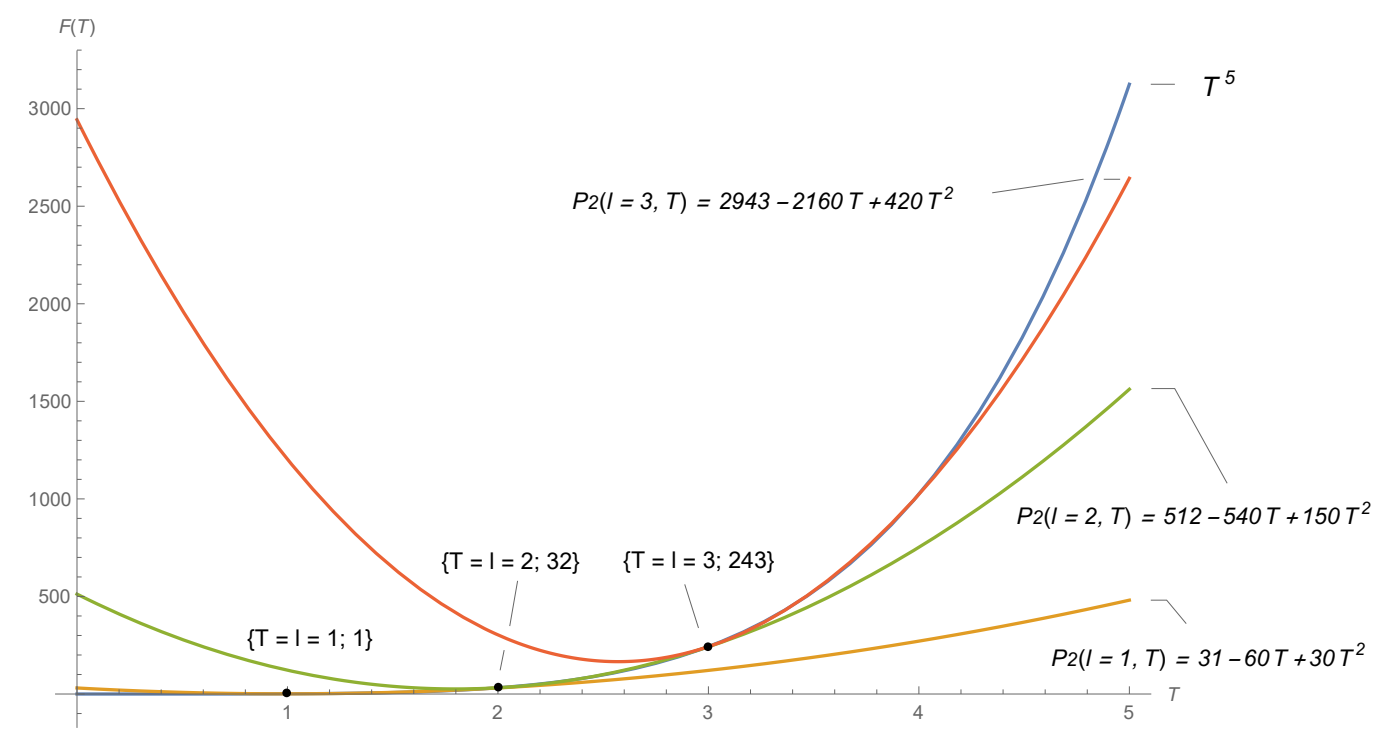

Figure 2. Approximations of monomial $T^{5}$ by polynomials $P_{2}(1, T), P_{2}(2, T), P_{2}(3, T)$ in some neighborhood of the point $\ell=1,2,3$, with $T \rightarrow \ell$ see 1.16 .

Similarly, finding the coefficients $A_{3,0}, A_{3,1}, A_{3,2}, A_{3,3}$ in $P_{3}(\ell, T)=\sum_{k=1}^{\ell} \sum_{j=0}^{3} A_{3, j} k^{j}(T-k)^{j}$, we get $A_{3,0}=1, A_{3,1}=0, A_{3,2}=-14, A_{3,3}=140$, therefore, for each $\ell=T \in \mathbb{N}$ :

$$
T^{7}=\sum_{1 \leq k \leq \ell} 140 k^{3}(T-k)^{3}-14 k^{2}(T-k)^{2}+1
$$

Below we show a few initial rows of the triangle, every $(\ell=T)$-th row sum starting from $k=1$ gives the terms of polynomial $P_{3}(\ell, T)=\sum_{k=1}^{\ell} \sum_{j=0}^{3} A_{3, j} k^{j}(T-k)^{j}=\sum_{k=1}^{\ell} 140 k^{3}(T-k)^{3}-14 k^{2}(T-$ $k)^{2}+1$, over $k$ in range $1 \leq k \leq \ell=T \in \mathbb{N}_{0}$. We review the triangle in context of the partial case of polynomial $P_{m}(\ell, T), m=3, \ell=T \in \mathbb{N}_{0}$ 


$$
\begin{aligned}
& \ell=T=0 \\
& \ell=T=1 \\
& 1 \\
& \ell=T=2 \\
& 1 \\
& \ell=T=3 \\
& 1 \\
& \ell=T=4 \\
& 1 \\
& \ell=T=5 \quad 1 \quad 8905 \\
& 3793 \\
& 1093 \\
& 1
\end{aligned}
$$

Table 3. Triangle generated by the polynomial $\sum_{j=0}^{3} A_{3, j} k^{j}(T-k)^{j}=140 k^{3}(T-k)^{3}-$ $14 k^{2}(T-k)^{2}+1,0 \leq k \leq \ell=T \in \mathbb{N}_{0}$, sequence A300785 in OEIS, 9. Summation of the $(\ell=T)$-th row terms from $k=1$ gives $P_{3}(T, T)=T^{7}$, see 1.1 .

As identity (1.1) holds, the sum of the $(T=\ell)$-th row terms of table 3 over $k$ from 1 to $T=\ell$ equals to $T^{2 m+1}, m=3$. For the case $P_{3}(\ell, T)$, the following polynomials $\sum_{k=1}^{\ell} \sum_{j=0}^{3} A_{3, j} k^{j}(T-$ $k)^{j}=\sum_{k=0}^{3}(-1)^{3-k} U_{3}(\ell, k) \cdot T^{k}$, for $\ell=1,2, \ldots, 10$ can be generated

$$
\begin{aligned}
P_{3}(\ell, T)= & \sum_{k=1}^{\ell} \sum_{j=0}^{3} A_{3, j} k^{j}(T-k)^{j}=\sum_{1 \leq k \leq \ell} 140 k^{3}(T-k)^{3}-14 k^{2}(T-k)^{2}+1=\sum_{k=0}^{3}(-1)^{3-k} U_{3}(\ell, k) \cdot T^{k} \\
& = \begin{cases}\ell=1: & -125+406 T-420 T^{2}+140 T^{3}=P_{3}(1, T) \\
\ell=2: & -9028+13818 T-7140 T^{2}+1260 T^{3}=P_{3}(2, T) \\
\ell=3: & -110961+115836 T-41160 T^{2}+5040 T^{3}=P_{3}(3, T) \\
\ell=4: & -684176+545860 T-148680 T^{2}+14000 T^{3}=P_{3}(4, T) \\
\ell=5: & -2871325+1858290 T-411180 T^{2}+31500 T^{3}=P_{3}(5, T) \\
\ell=6: & -9402660+5124126 T-955500 T^{2}+61740 T^{3}=P_{3}(6, T) \\
\ell=7: & -25872833+12182968 T-1963920 T^{2}+109760 T^{3}=P_{3}(7, T) \\
\ell=8: & -62572096+25945416 T-3684240 T^{2}+181440 T^{3}=P_{3}(8, T) \\
\ell=9: & -136972701+50745870 T-6439860 T^{2}+283500 T^{3}=P_{3}(9, T) \\
\ell=10: & -276971300+92745730 T-10639860 T^{2}+423500 T^{3}=P_{3}(10, T)\end{cases} \\
= & T^{7} \text { as } T \rightarrow \ell .
\end{aligned}
$$

The coefficients $U_{3}(T, k), 0 \leq k \leq 3$ in (1.17) are terms of the sequence A316387, [18]. Above polynomials are approximations of monomial $T^{7}$ in some neighborhood of the point $T \rightarrow \ell$. The following graph shows corresponding polynomials $\sum_{k=0}^{3}(-1)^{3-k} U_{3}(\ell, k) \cdot T^{k}, T=1,2,3$ and the $T^{7}$ curve 


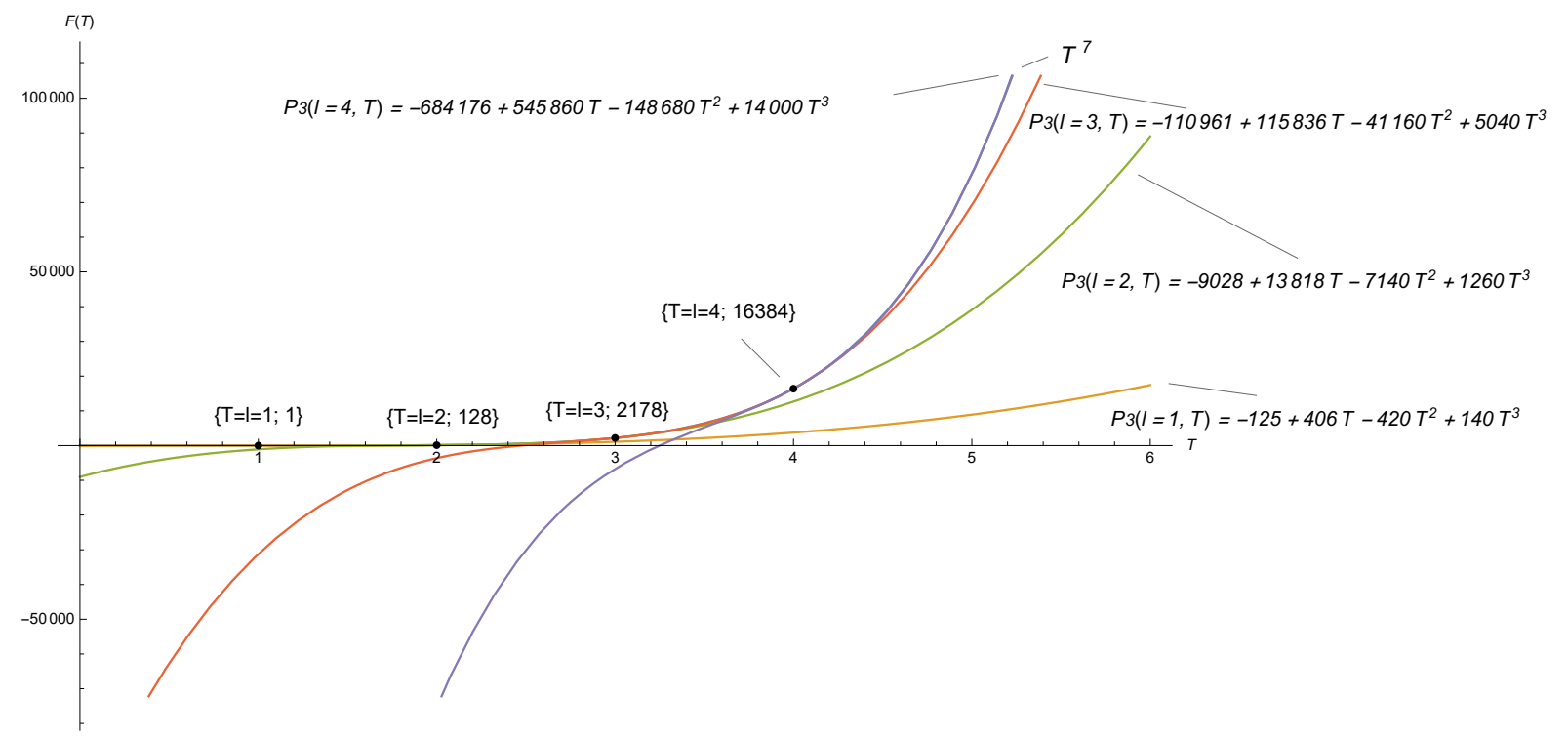

Figure 3. Approximations of monomial $T^{7}$ by polynomials $P_{3}(1, T), P_{3}(2, T), P_{3}(3, T)$ in some neighborhood of the point $\ell=1,2,3$, with $T \rightarrow \ell$, see (1.17).

Now, let generalise the the identity $P_{m}(T, T)=T^{2 m+1}, T \in \mathbb{N}$ for every integer $m \geq 0$, as we have already discussed the cases $m=1,2,3$. Firstly, let's find a recurrence formula for coefficients $A_{m, j}, 0 \leq j \leq m$, see definition (1.1). To reach a recurrent formula of $A_{m, j}, m \geq 0$, first let's fix the unused values of $A_{m, j}=0$, for $j<0$ or $j>m$, so we don't need to consider the summation range for $j$. Let's rewrite the polynomial $P_{m}(\ell, T)$ again

$$
P_{m}(\ell, T)=\sum_{k=1}^{\ell} \sum_{j=0}^{m} A_{m, j} k^{j}(T-k)^{j} \equiv \sum_{k=0}^{m}(-1)^{m-k} U_{m}(\ell, k) \cdot T^{k},
$$

Since the symmetry holds

$$
D_{m}(T, k):=\sum_{j=0}^{m} A_{m, j} k^{j}(T-k)^{j}, D_{m}(T, k)=D_{m}(T-k, k)
$$

We can rewrite 1.18 as follows

$$
\sum_{k=1}^{\ell} \sum_{j=0}^{m} A_{m, j} k^{j}(T-k)^{j}=\sum_{k=0}^{\ell-1} \sum_{j=0}^{m} A_{m, j} k^{j}(T-k)^{j}
$$

By expanding $(T-k)^{j}$ in r.h.s of 1.19$)$ and using Faulhaber's formula [1], the result is

$$
\begin{aligned}
& \sum_{k=0}^{\ell-1}(T-k)^{j} k^{j}=\sum_{k=0}^{\ell-1} \sum_{i}\left(\begin{array}{c}
j \\
i
\end{array}\right) T^{j-i}(-1)^{i} k^{i+j} \\
& =\sum_{i}\left(\begin{array}{c}
j \\
i
\end{array}\right) T^{j-i} \frac{(-1)^{i}}{i+j+1}\left[\sum_{t}\left(\begin{array}{c}
i+j+1 \\
t
\end{array}\right) B_{t} \ell^{i+j+1-t}-B_{i+j+1}\right] \\
& =\underbrace{\sum_{i, t}\left(\begin{array}{c}
j \\
i
\end{array}\right) \frac{(-1)^{i}}{i+j+1}\left(\begin{array}{c}
i+j+1 \\
t
\end{array}\right) B_{t} T^{j-i} \ell^{i+j+1-t}}_{(\star)}-\underbrace{\sum_{i}\left(\begin{array}{c}
j \\
i
\end{array}\right) \frac{(-1)^{i}}{i+j+1} B_{i+j+1} T^{j-i}}_{(\diamond)}
\end{aligned}
$$

where $B_{t}$ are Bernoulli numbers [14]. Now, we notice that

$$
\sum_{i}\left(\begin{array}{l}
j \\
i
\end{array}\right) \frac{(-1)^{i}}{i+j+1}\left(\begin{array}{c}
i+j+1 \\
t
\end{array}\right)= \begin{cases}\frac{1}{(2 j+1)\left(\begin{array}{c}
2 j \\
j
\end{array}\right)}, & \text { if } t=0 \\
\frac{(-1)^{j}}{t}\left(\begin{array}{c}
j \\
2 j-t+1
\end{array}\right), & \text { if } t>0 .\end{cases}
$$


In particular, the last sum is zero for $0<t \leq j$. Now, by substituting to the $(\star)$ part of $(1.20)$ the result of (1.21), we have

$$
\begin{aligned}
\sum_{i, t}\left(\begin{array}{l}
j \\
i
\end{array}\right) \frac{(-1)^{i}}{i+j+1}\left(\begin{array}{c}
i+j+1 \\
t
\end{array}\right) B_{t} T^{j-i} \ell^{i+j+1-t} & =\frac{1}{(2 j+1)\left(\begin{array}{c}
2 j \\
j
\end{array}\right)} T^{j} \ell^{j+1} \\
& +\sum_{t>0} \frac{(-1)^{j}}{t}\left(\begin{array}{c}
j \\
2 j-t+1
\end{array}\right) B_{t} T^{j-i} \ell^{i+j+1-t}
\end{aligned}
$$

By means of 1.22 , expression 1.20 takes the form

$$
\begin{aligned}
\sum_{k=0}^{\ell-1}(T-k)^{j} k^{j} & =\underbrace{\frac{1}{(2 j+1)\left(\begin{array}{c}
(2 j \\
j
\end{array}\right)} T^{j} \ell^{j+1}+\sum_{t>0} \frac{(-1)^{j}}{t}\left(\begin{array}{c}
j \\
2 j-t+1
\end{array}\right) B_{t} T^{j-i} \ell^{i+j+1-t}}_{(\star)} \\
& -\underbrace{\sum_{i}\left(\begin{array}{c}
j \\
i
\end{array}\right) \frac{(-1)^{i}}{i+j+1} B_{i+j+1} T^{j-i}}_{(\diamond)}
\end{aligned}
$$

We have to remember that if the sum over some variable $i$ contains $\left(\begin{array}{l}j \\ i\end{array}\right)$, then instead of limiting its summation range to $i \in[0, j]$, we can let $i \in[-\infty,+\infty]$ since $\left(\begin{array}{l}j \\ i\end{array}\right)=0$ for $i$ outside the range $i \in[0, j]$ (i.e., when $i<0$ or $i>j$ ). It's much easier to review such sum as sum from $-\infty$ to $+\infty$ (unless specified otherwise), where only a finite number of terms are nonzero, this fact is also discussed in [12]. Therefore, we haven't shown detailed bounds of summation in above derivation. Now, we keep our attention on (1.23). To combine or cancel identical terms across the two sums in (1.23) more easily, let introduce $\kappa=2 j+1-t$ to $(\star)$ and $\kappa=j-i$ to $(\diamond)$, respectively

$$
\begin{aligned}
& \sum_{k=0}^{\ell-1}(T-k)^{j} k^{j}=\frac{1}{(2 j+1)\left(\begin{array}{c}
2 j \\
j
\end{array}\right)} T^{j} \ell^{j+1}+\sum_{\kappa} \frac{(-1)^{j}}{2 j+1-\kappa}\left(\begin{array}{l}
j \\
\kappa
\end{array}\right) B_{2 j+1-\kappa} T^{j-i} \ell^{\kappa+i-j} \\
& -\sum_{\kappa}\left(\begin{array}{l}
j \\
\kappa
\end{array}\right) \frac{(-1)^{j-\kappa}}{2 j+1-\kappa} B_{2 j+1-\kappa} T^{\kappa}
\end{aligned}
$$

Let be $T=\ell$ in $(1.24)$, thus

$$
\begin{aligned}
& \sum_{k=0}^{T-1}(T-k)^{j} k^{j}=\frac{1}{(2 j+1)\left(\begin{array}{c}
2 j \\
j
\end{array}\right)} T^{2 j+1}+\sum_{\kappa} \frac{(-1)^{j}}{2 j+1-\kappa}\left(\begin{array}{l}
j \\
\kappa
\end{array}\right) B_{2 j+1-\kappa} T^{\kappa} \\
& -\sum_{\kappa}\left(\begin{array}{l}
j \\
\kappa
\end{array}\right) \frac{(-1)^{j-\kappa}}{2 j+1-\kappa} B_{2 j+1-\kappa} T^{\kappa} \\
& =\frac{1}{(2 j+1)\left(\begin{array}{c}
2 j \\
j
\end{array}\right)} T^{2 j+1}+2 \sum_{\text {odd } \kappa} \frac{(-1)^{j}}{2 j+1-\kappa}\left(\begin{array}{l}
j \\
\kappa
\end{array}\right) B_{2 j+1-\kappa} T^{\kappa} .
\end{aligned}
$$

Now, using the definition of $A_{m, j}$,

$$
\sum_{k=0}^{\ell-1} \sum_{j} A_{m, j} k^{j}(T-k)^{j}=T^{2 m+1}, \text { as } \ell=T \in \mathbb{N},
$$

From 1.25 we can obtain the following identity for polynomials in $T$

$$
\begin{aligned}
& \sum_{j} A_{m, j} \frac{1}{(2 j+1)\left(\begin{array}{c}
2 j \\
j
\end{array}\right)} T^{2 j+1}+2 \sum_{j, \text { odd } \kappa} A_{m, j}\left(\begin{array}{l}
j \\
\kappa
\end{array}\right) \frac{(-1)^{j}}{2 j+1-\kappa} B_{2 j+1-\kappa} T^{\kappa} \\
& \equiv n^{2 m+1}, \text { as } T \rightarrow \ell .
\end{aligned}
$$

Taking the coefficient of $n^{2 j+1}$ in the above expression, we get $A_{m, m}=(2 m+1)\left(\begin{array}{c}2 m \\ m\end{array}\right)$, and taking the coefficient of $x^{2 d+1}$ for an integer $d$ in the range $m / 2 \leq d<m$ we get $A_{m, d}=0$. Taking the 
coefficient of $n^{2 d+1}$ in 1.26 for $m / 4 \leq d<m / 2$, we get

i.e

$$
A_{m, d} \frac{1}{(2 d+1)\left(\begin{array}{c}
2 d \\
d
\end{array}\right)}+2(2 m+1)\left(\begin{array}{c}
2 m \\
m
\end{array}\right)\left(\begin{array}{c}
m \\
2 d+1
\end{array}\right) \frac{(-1)^{m}}{2 m-2 d} B_{2 m-2 d}=0
$$

$$
A_{m, d}=(-1)^{m-1} \frac{(2 m+1) !}{d ! d ! m !(m-2 d-1) !} \frac{1}{m-d} B_{2 m-2 d} .
$$

Continuing similarly, we can express $A_{m, j}$ for each integer $j$ in the range $m / 2^{s+1} \leq j<m / 2^{s}$ (iterating consecutively $s=1,2, \ldots$ ) via the previously determined values of $A_{m, d}, d<j$ as follows

$$
A_{m, j}=(2 j+1)\left(\begin{array}{c}
2 j \\
j
\end{array}\right) \sum_{d=2 j+1}^{m} A_{m, d}\left(\begin{array}{c}
d \\
2 j+1
\end{array}\right) \frac{(-1)^{d-1}}{d-j} B_{2 d-2 j}
$$

The same formula holds also for $m=0$. Note that the $m$ in above sum must satisfy $m \geq 2 j+1$ to return a nonzero term $A_{m, j}$.

Definition 1.27. We define here a generating function of sequence of coefficients $A_{m, j}$ as follows

$$
A_{m, j}:= \begin{cases}0, & \text { if } j<0 \text { or } j>m \\
(2 j+1)\left(\begin{array}{c}
2 j \\
j
\end{array}\right) \sum_{d=2 j+1}^{m} A_{m, d}\left(\begin{array}{c}
d \\
2 j+1
\end{array}\right) \frac{(-1)^{d-1}}{d-j} B_{2 d-2 j}, & \text { if } 0 \leq j<m \\
(2 j+1)\left(\begin{array}{c}
2 j \\
j
\end{array}\right), & \text { if } j=m\end{cases}
$$

Five initial rows of the triangle generated by $A_{m, j}, j \geq 0,0 \leq j \leq m$ are

$$
\begin{aligned}
& m=0 \quad 1 \\
& m=1 \quad 1 \quad 6 \\
& \begin{array}{llll}
m=2 & 1 & 0 & 30
\end{array} \\
& \begin{array}{lllll}
m=3 & 1 & -14 & 0 & 140
\end{array} \\
& \begin{array}{llllll}
m=4 & 1 & -120 & 0 & 0 & 630
\end{array} \\
& \begin{array}{lllllll}
m=5 & 1 & -1386 & 660 & 0 & 0 & 2772
\end{array}
\end{aligned}
$$

Table 4. Triangle generated by $A_{m, j}, j \geq 0,0 \leq j \leq m$, see definition (1.27)

Note that starting from row $m \geq 11$ the terms of the table 4 consist of fractional numbers, for example, $A_{11,1}=800361655623.6$. One can find a complete list of the numerators and denominators of $A_{m, j}$ in OEIS under the identifiers A302971 and A304042, respectively, see [17], 18]. Note that

$$
\sum_{j} A_{m, j}=2^{2 m+1}-1 .
$$

As we have found a recurrence for $A_{m, j}$ coefficients, let's back to the identity (1.1)

$$
\sum_{k=1}^{\ell} \sum_{j=0}^{m} A_{m, j} k^{j}(T-k)^{j} \equiv \sum_{k=0}^{m}(-1)^{m-k} U_{m}(\ell, k) \cdot T^{k}
$$

to find a recurrence for coefficients $U_{m}(\ell, k)$ for every $m \geq 1$. To understand the nature of the coefficients $U_{m}(T, k)$, express the polynomials $U_{m}(\ell, k)$ in terms of $A_{m, j}$ and Bernoulli numbers. To do so, let's expand the binomial $(T-k)^{j}$ in the l.h.s. of 1.1 and change of the order of summation:

$$
\begin{aligned}
\sum_{k=1}^{\ell} \sum_{j=0}^{m} A_{m, j} k^{j}(T-k)^{j} & =\sum_{k=1}^{\ell} \sum_{j=0}^{m} A_{m, j} k^{j} \sum_{t=0}^{j}\left(\begin{array}{l}
j \\
t
\end{array}\right) T^{t}(-1)^{j-t} k^{j-t} \\
& =\sum_{t=0}^{m} T^{t} \sum_{k=1}^{\ell} \sum_{j=t}^{m}\left(\begin{array}{l}
j \\
t
\end{array}\right) A_{m, j} k^{2 j-t}(-1)^{j-t} .
\end{aligned}
$$


Now, taking the coefficient of $T^{t}$ in 1.28 gives:

$$
U_{m}(\ell, t)=(-1)^{m} \sum_{k=1}^{\ell} \sum_{j=t}^{m}\left(\begin{array}{l}
j \\
t
\end{array}\right) A_{m, j} k^{2 j-t}(-1)^{j} .
$$

From this formula it may be not immediately clear why as $T \rightarrow \ell$ the $U_{m}(\ell, t)$ represent polynomials in $T$. However, this can be seen if we change the summation order again and use Faulhaber's formula to obtain:

$$
U_{m}(T, t)=(-1)^{m} \sum_{j=t}^{m}\left(\begin{array}{l}
j \\
t
\end{array}\right) A_{m, j} \frac{(-1)^{j}}{2 j-t+1} \sum_{l=0}^{2 j-t}\left(\begin{array}{c}
2 j-t+1 \\
l
\end{array}\right) B_{l} T^{2 j-t+1-l} .
$$

Introducing $\kappa=2 j-t+1-l$, to 1.29 we further get the formula:

$$
U_{m}(T, t)=(-1)^{m} \sum_{k=1}^{2 j-t+1} T^{k} \sum_{j=t}^{m}\left(\begin{array}{l}
j \\
t
\end{array}\right) A_{m, j} \frac{(-1)^{j}}{2 j-t+1}\left(\begin{array}{c}
2 j-t+1 \\
\kappa
\end{array}\right) B_{2 j-t+1-\kappa},
$$

which allows easily compute the coefficient of $T^{k}$ in $U_{m}(T, t)$ for each $\kappa$. In above formulae we assume that $B_{1}=+\frac{1}{2}$.

1.1. Error of approximation. Generally, the monomial $T^{2 m+1}, \ell, m \in \mathbb{N}, T \in \mathbb{R}$ could be approximated by (1.1) as follows

$$
\lim _{T \rightarrow \ell}\left[\sum_{k=1}^{\ell} \sum_{j=0}^{m} A_{m, j} k^{j}(T-k)^{j}\right]=\lim _{T \rightarrow \ell}\left[\sum_{k=0}^{m}(-1)^{m-k} U_{m}(\ell, k) \cdot T^{k}\right]=T^{2 m+1} .
$$

In this subsection we arrange the tables with values of corresponding polynomials $P_{m}(\ell, T)$ and monomials $T^{2 m+1}$ to rate the quality of the approximation of $T^{2 m+1}, m \geq 0$ by (1.1). We begin from the case $m=1$ and $T=3$, therefore, the approximating polynomials is $P_{1} 3, T=-81+36 T$. By solving the equation $T^{3}-36 T+81=0$ we receive the following roots $T_{1}=3, T_{2}=3.90833$, it follows that our polynomial approximates the monomial $T^{2 m+1}$ in neighborhood of $T_{1}$ and $T_{2}$, the following table contains the values of $P_{1}(3, T)$ and $T^{3}$ in neighborhood of the root $T_{1}=3$

\begin{tabular}{|c|c|c|}
\hline$T$ & $F(T)=T^{3}$ & $P_{1}(3, T)=-81+36 T$ \\
\hline 2.95 & 25.67238 & 25.2 \\
\hline 2.96 & 25.93434 & 25.56 \\
\hline 2.97 & 26.19807 & 25.92 \\
\hline 2.98 & 26.46359 & 26.28 \\
\hline 2.99 & 26.73090 & 26.64 \\
\hline$\underline{3.00}$ & $\underline{27.00000}$ & $\underline{27}$ \\
\hline 3.01 & 27.27090 & 27.36 \\
\hline 3.02 & 27.54361 & 27.72 \\
\hline 3.03 & 27.81813 & 28.08 \\
\hline 3.04 & 28.09446 & 28.44 \\
\hline 3.05 & 28.37263 & 28.8 \\
\hline
\end{tabular}

Table 5. Table of values of $F(T)=T^{3}$ and $P_{1}(3, T)$ in neighborhood of $T_{1}=3$.

Below we show graphically the intersection of of $F(T)=T^{3}$ and $P_{1}(3, T)$ 


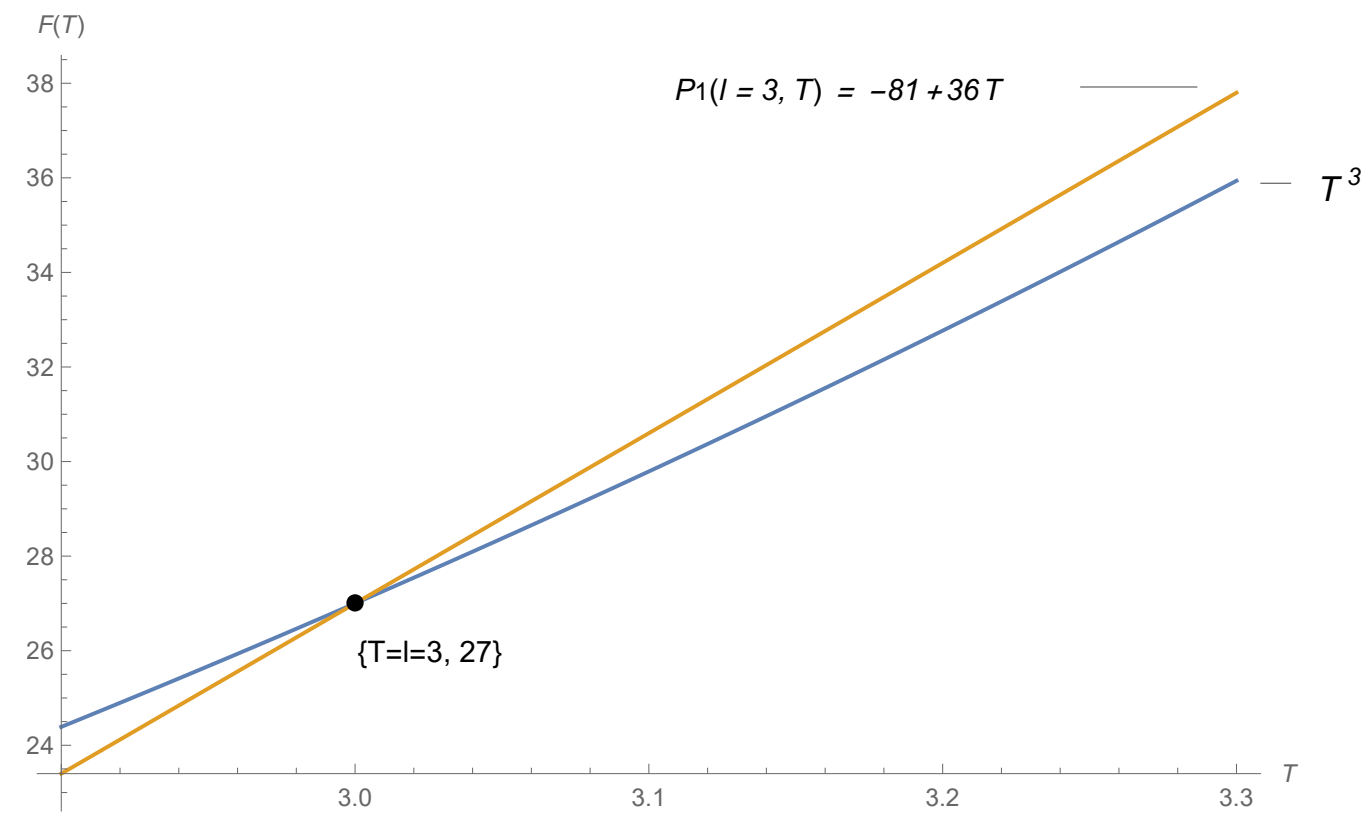

Figure 4. Intersection of $F(T)=T^{3}$ and $P_{1}(3, T)$ in point $T_{1}=3$.

As it is mentioned above the equation $T^{3}-36 T+81=0$ has two roots $T_{1}=3, T_{2}=3.90833$, let's discuss the case $T_{2}=3.90833$, the following table represents the values of $P_{1}(3, T)$ and $T^{3}$ in neighborhood of the root $T_{2}=3.90833$

\begin{tabular}{|c|c|c|}
\hline$T$ & $F(T)=T^{3}$ & $P_{1}(3, T)=-81+36 T$ \\
\hline 3.86000 & 57.51246 & 57.96 \\
\hline 3.87000 & 57.96060 & 58.32 \\
\hline 3.88000 & 58.41107 & 58.68 \\
\hline 3.89000 & 58.86387 & 59.04 \\
\hline 3.90000 & 59.31900 & 59.4 \\
\hline$\underline{3.90833}$ & $\underline{59.69991}$ & $\underline{59.69988}$ \\
\hline 3.92000 & 60.23629 & 60.12 \\
\hline 3.93000 & 60.69846 & 60.48 \\
\hline 3.94000 & 61.16298 & 60.84 \\
\hline 3.95000 & 61.62988 & 61.2 \\
\hline 3.96000 & 62.09914 & 61.56 \\
\hline
\end{tabular}

Table 6. Table of values of $F(T)=T^{3}$ and $P_{1}(3, T)$ in neighborhood of $T_{1}=3$.

Below we show graphically the intersection of $F(T)=T^{3}$ and $P_{1}(3, T)$ 


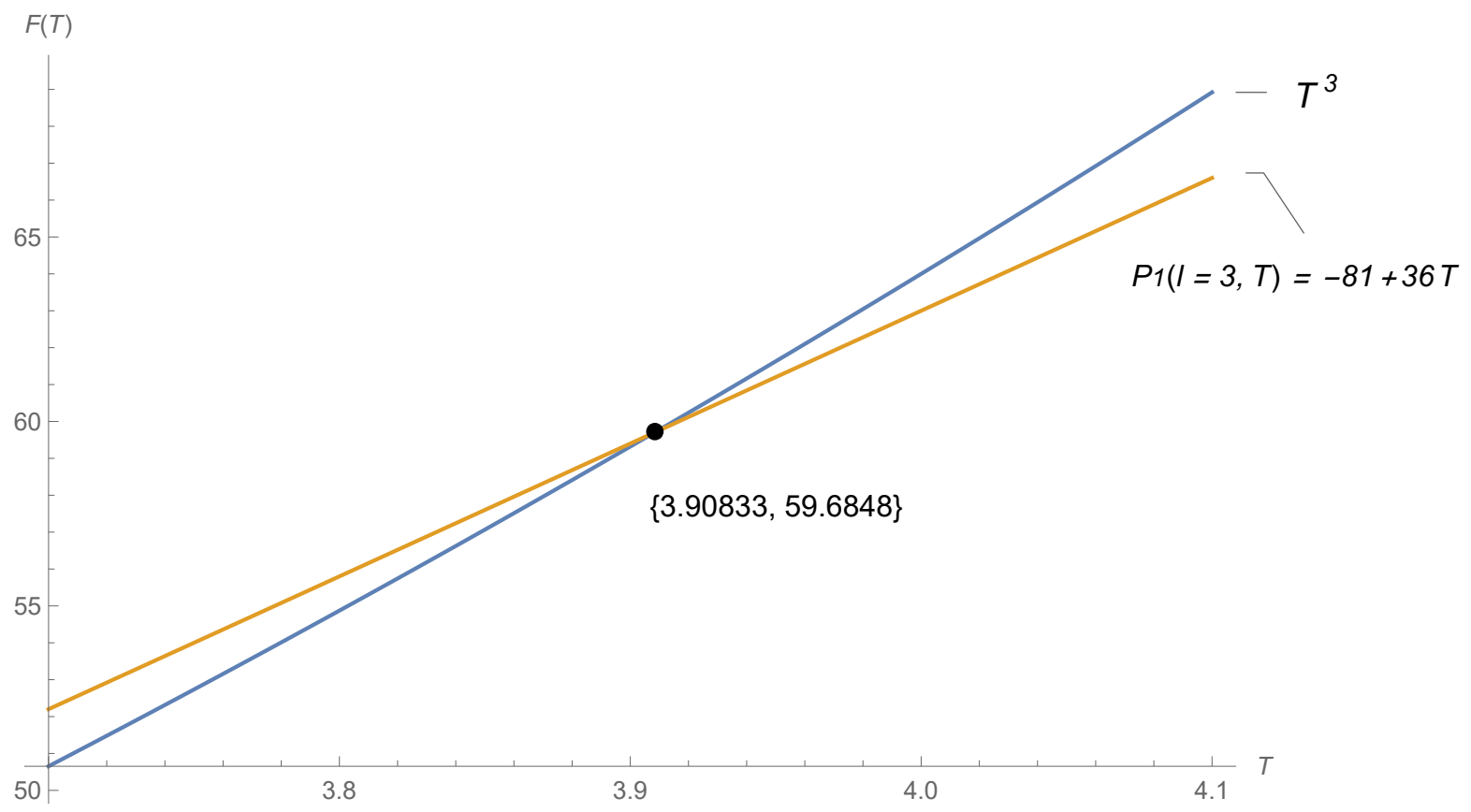

Figure 5. Intersection of $F(T)=T^{3}$ and $P_{1}(3, T)$ in point $T_{2}=3.90833$.

Next, let's review the case $m=2$ and $T=3$, the approximating polynomial is $P_{2}(3, T)=$ $2943-2160 T+420 T^{2}$. By solving the equation $T^{5}-\left(2943-2160 T+420 T^{2}\right)=0$ we receive the following roots $T_{1}=3, T_{2}=3.40551, T_{3}=3.98704$, it follows that our polynomial approximates the monomial $T^{2 m+1}$ in neighborhood of $T_{1}, T_{2}$ and $T_{3}$, the following tables contain the values of $P_{2}(3, T)$ and $T^{5}$ in neighborhood of the roots $T_{1}=3, T_{2}$ and $T_{3}$

\begin{tabular}{|c|c|c|}
\hline$T$ & $F(T)=T^{5}$ & $P_{2}(3, T)=2943-2160 T+420 T^{2}$ \\
\hline 2.95 & 223.41384 & 226.05000 \\
\hline 2.96 & 227.22628 & 229.27200 \\
\hline 2.97 & 231.09058 & 232.57800 \\
\hline 2.98 & 235.00728 & 235.96800 \\
\hline 2.99 & 238.97691 & 239.44200 \\
\hline$\underline{3.00}$ & $\underline{243.00000}$ & $\underline{243.00000}$ \\
\hline 3.01 & 247.07709 & 246.64200 \\
\hline 3.02 & 251.20872 & 250.36800 \\
\hline 3.03 & 255.39544 & 254.17800 \\
\hline 3.04 & 259.63780 & 258.07200 \\
\hline 3.05 & 263.93634 & 262.05000 \\
\hline
\end{tabular}

Table 7. Table of values of $F(T)=T^{5}$ and $P_{2}(3, T)$ in neighborhood of $T_{1}=3$.

Below we show graphically the intersection of $F(T)=T^{5}$ and $P_{2}(3, T)$ 


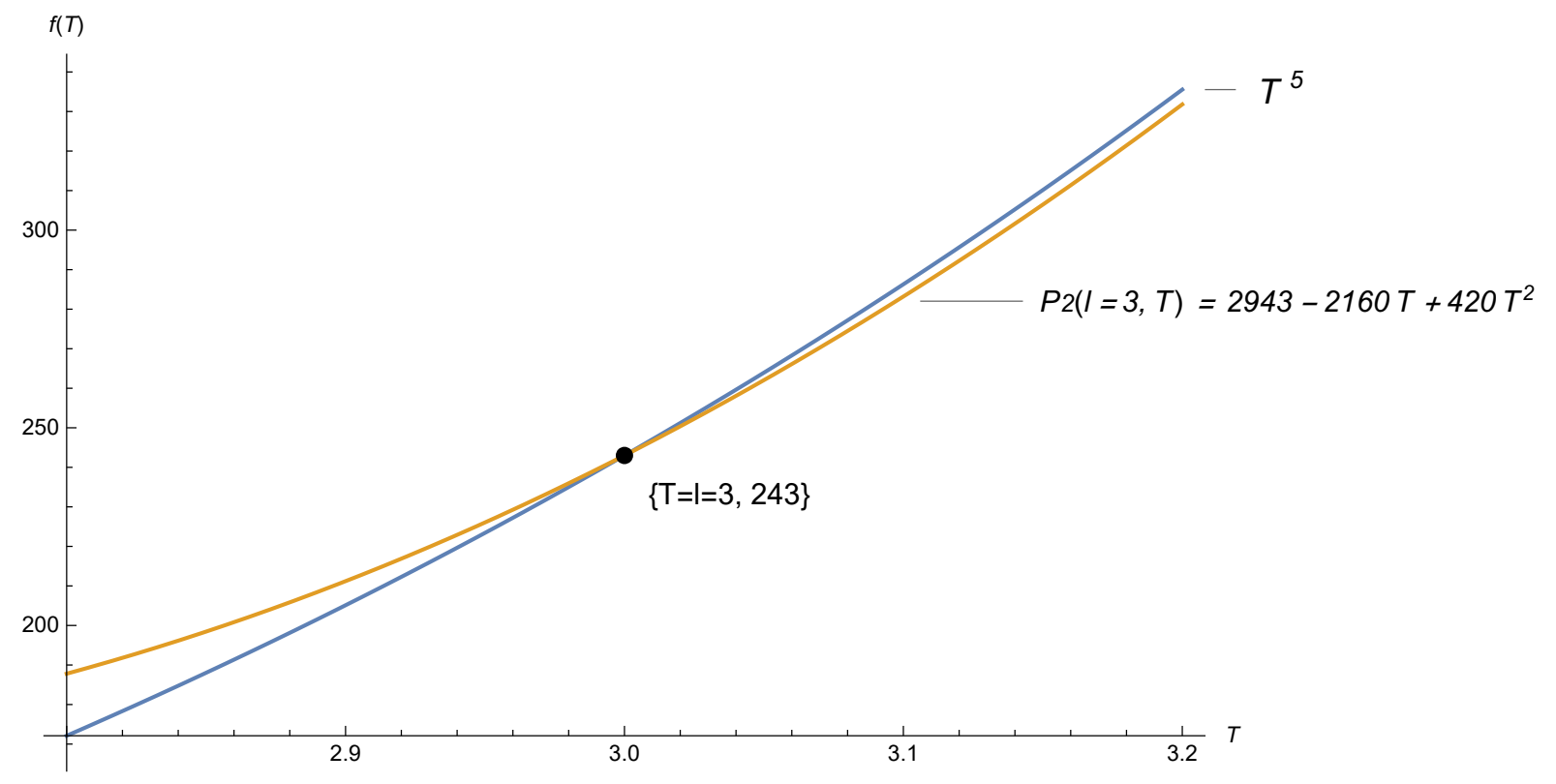

Figure 6. Intersection of $F(T)=T^{5}$ and $P_{2}(3, T)$ in point $T_{1}=3$.

Next, let's review the values of $F(T)=T^{5}$ and $P_{2}(3, T)$ in the neighborhood of the root $T_{2}=$ 3.40551 as follow table shows

\begin{tabular}{|c|c|c|}
\hline$T$ & $F(T)=T^{5}$ & $P_{2}(3, T)=2943-2160 T+420 T^{2}$ \\
\hline 3.35000 & 421.91410 & 420.45000 \\
\hline 3.36000 & 428.24903 & 427.03200 \\
\hline 3.37000 & 434.65983 & 433.69800 \\
\hline 3.38000 & 441.14717 & 440.44800 \\
\hline 3.39000 & 447.71175 & 447.28200 \\
\hline$\underline{3.40551}$ & 458.04780 & 458.04771 \\
\hline 3.41000 & 461.07534 & 461.20200 \\
\hline 3.42000 & 467.87574 & 468.28800 \\
\hline 3.43000 & 474.75615 & 475.45800 \\
\hline 3.44000 & 481.71727 & 482.71200 \\
\hline 3.45000 & 488.75980 & 490.05000 \\
\hline
\end{tabular}

Table 8. Table of values of $F(T)=T^{5}$ and $P_{2}(3, T)$ in neighborhood of $T_{2}=3.40551$.

Below we show graphically the intersection of $F(T)=T^{5}$ and $P_{2}(3, T)$ in point $T_{2}=3.40551$ 


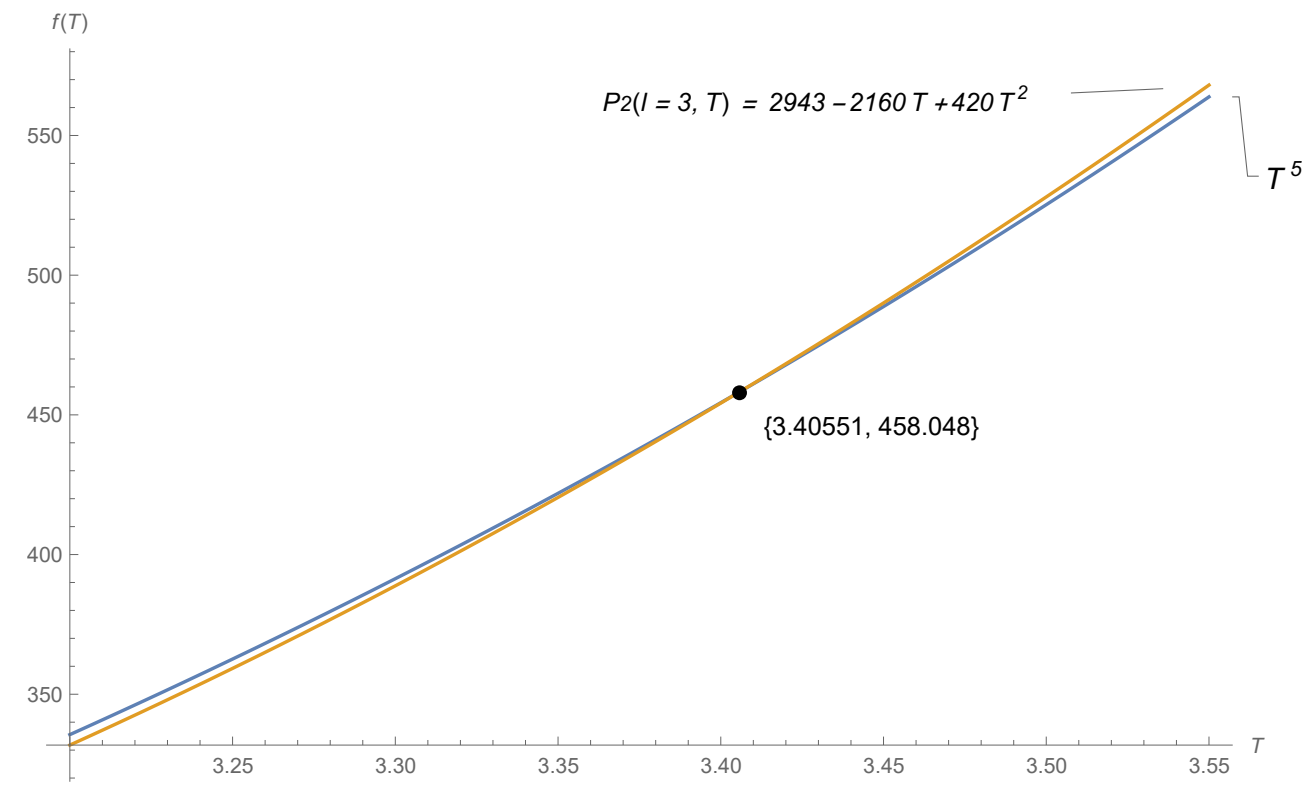

Figure 7. Intersection of $F(T)=T^{5}$ and $P_{2}(3, T)$ in point $T_{2}=3.90833$.

Now, let's review the values of $F(T)=T^{5}$ and $P_{2}(3, T)$ in the neighborhood of the root $T_{2}=$ 3.90833 as follow table shows

\begin{tabular}{|c|c|c|}
\hline$T$ & $F(T)=T^{5}$ & $P_{2}(3, T)=2943-2160 T+420 T^{2}$ \\
\hline 3.93000 & 937.48160 & 941.05800 \\
\hline 3.94000 & 949.46970 & 952.51200 \\
\hline 3.95000 & 961.58012 & 964.05000 \\
\hline 3.96000 & 973.81381 & 975.67200 \\
\hline 3.97000 & 986.17170 & 987.37800 \\
\hline 3.98000 & 998.65472 & 999.16800 \\
\hline$\underline{3.98704}$ & $\underline{1007.51835}$ & $\underline{1007.51854}$ \\
\hline 4.00000 & 1024.00000 & 1023.00000 \\
\hline 4.01000 & 1036.86416 & 1035.04200 \\
\hline 4.02000 & 1049.85728 & 1047.16800 \\
\hline 4.03000 & 1062.98034 & 1059.37800 \\
\hline
\end{tabular}

Table 9. Table of values of $F(T)=T^{5}$ and $P_{2}(3, T)$ in neighborhood of $T_{3}=3.98704$.

Below we show graphically the intersection of $F(T)=T^{5}$ and $P_{2}(3, T)$ in point $T_{3}=3.98704$ 


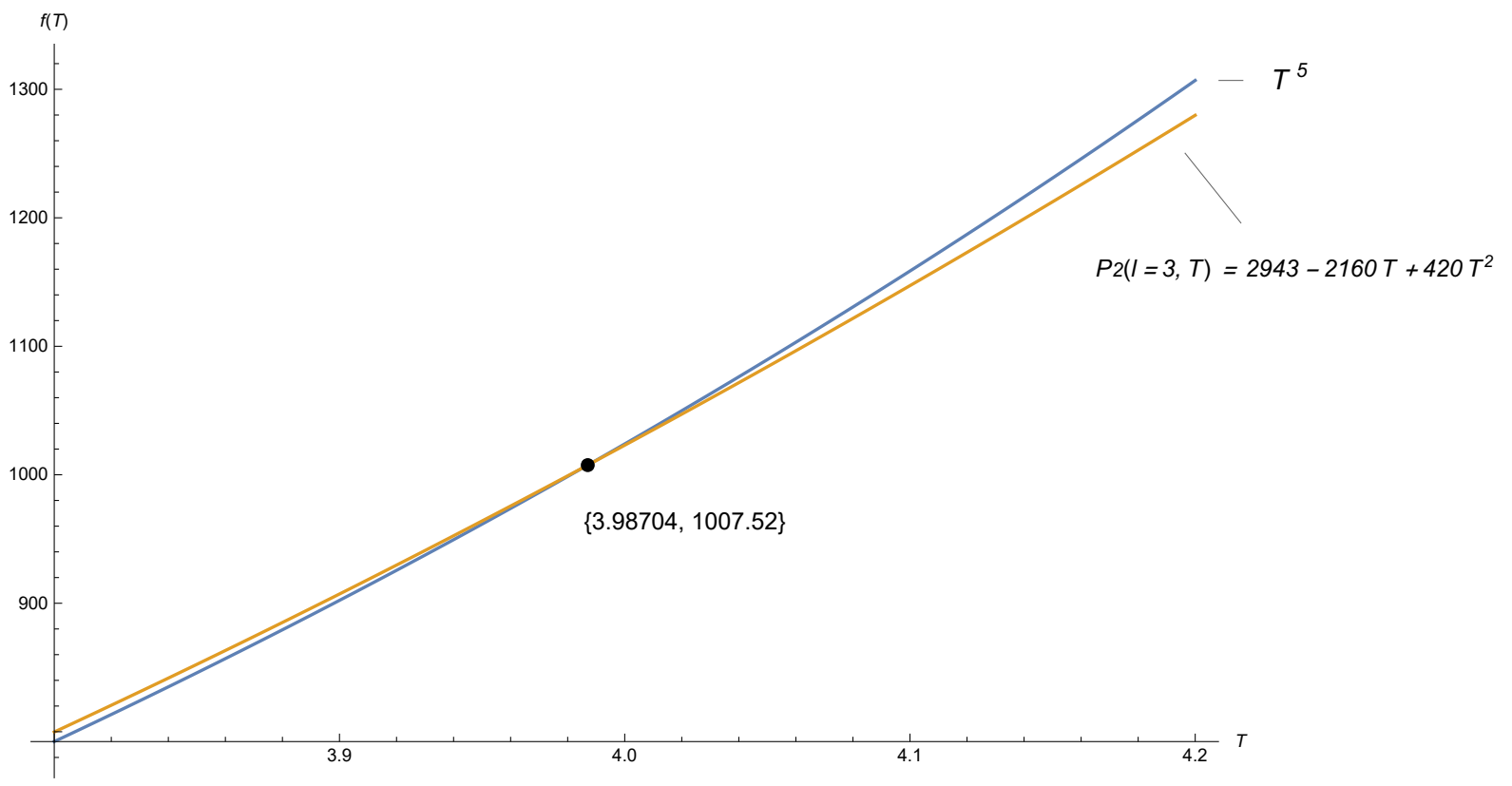

Figure 8. Intersection of $F(T)=T^{5}$ and $P_{2}(3, T)$ in point $T_{3}=3.98704$.

Now, let's keep our attention to the case $m=3$ and $\ell=2$, the approximating polynomial is $P_{3}(2, T)=-9028+13818 T-7140 T^{2}+1260 T^{3}$. By solving the equation $T^{7}-(-9028+13818 T-$ $\left.7140 T^{2}+1260 T^{3}\right)=0$ we receive the following roots $T_{1}=2, T_{2}=2.8605, T_{3}=2.98955$, it follows that our polynomial approximates the monomial $T^{7}$ in neighborhood of $T_{1}, T_{2}$ and $T_{3}$, the following tables contain the values of $P_{2}(3, T)$ and $T^{5}$ in neighborhood of the roots $T_{1}, T_{2}$ and $T_{3}$, note that we have arranged the roots $T_{2}$ and $T_{3}$ in single table

\begin{tabular}{|c|c|c|}
\hline$T$ & $F(T)=T^{7}$ & $P_{3}(2, T)=-9028+13818 T-7140 T^{2}+1260 T^{3}$ \\
\hline 1.96 & 111.12007 & 113.47136 \\
\hline 1.97 & 115.14990 & 117.00398 \\
\hline 1.98 & 119.30436 & 120.59792 \\
\hline 1.99 & 123.58664 & 124.26074 \\
\hline$\underline{2.00}$ & $\underline{128.00000}$ & $\underline{128}$ \\
\hline 2.01 & 132.54776 & 131.82326 \\
\hline 2.02 & 137.23333 & 135.73808 \\
\hline 2.03 & 142.06015 & 143.875202 \\
\hline 2.04 & 147.03177 & 148.1075 \\
\hline 2.05 & 152.15178 & 152.46416 \\
\hline 2.06 & 157.42385 & $1)=-9028+13818 T-710 T^{2}+12$ \\
\hline
\end{tabular}

Table 10. Table of values of $F(T)=T^{7}$ and $P_{3}(2, T)=-9028+13818 T-7140 T^{2}+1260 T^{3}$ in neighborhood of $T_{1}=2$.

Below we arrange a plot of intersection of $F(T)=T^{7}$ and $P_{3}(2, T)=-9028+13818 T-7140 T^{2}+$ $1260 T^{3}$ in $T_{1}=2$ 


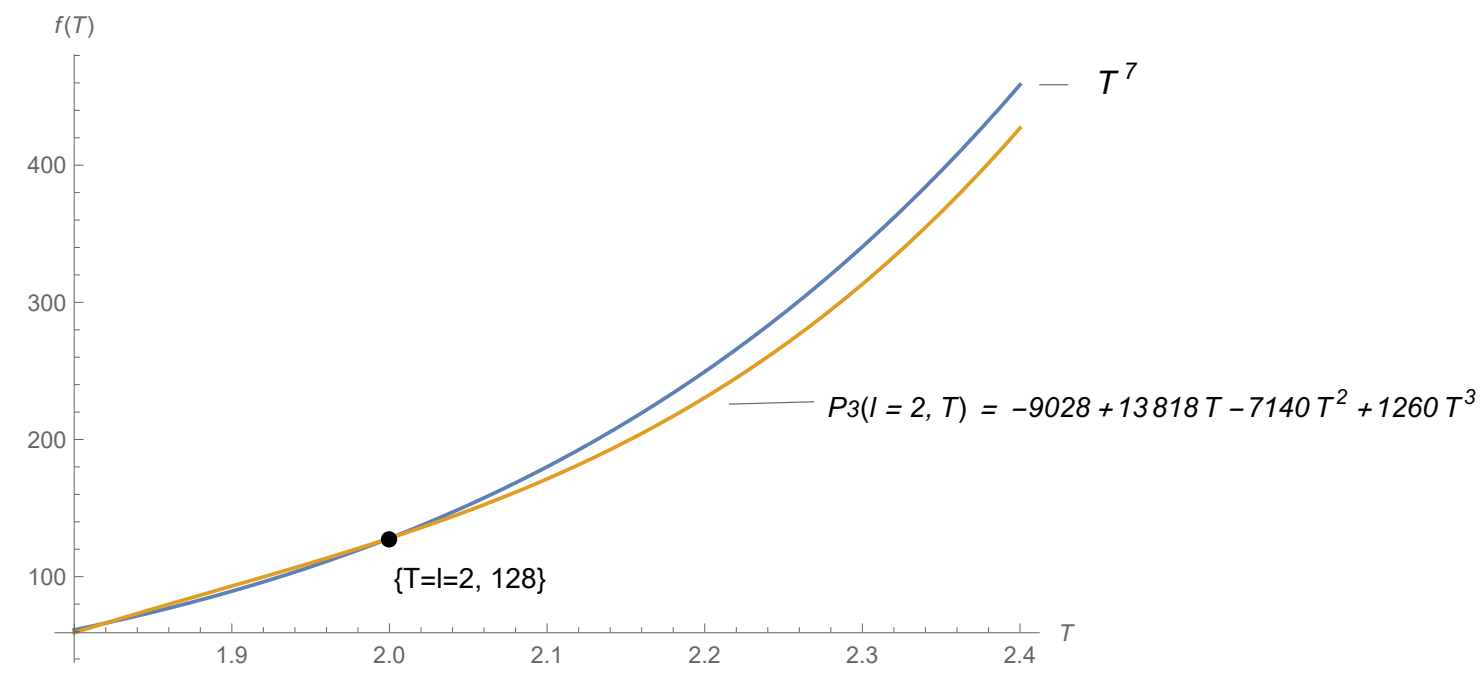

Figure 9. Intersection of $F(T)=T^{7}$ and $P_{2}(2, T)$ in point $T_{1}=2$.

Let's show the values of the $F(T)=T^{7}$ and polynomial $P_{3}(2, T)=-9028+13818 T-7140 T^{2}+$ $1260 T^{3}$ in neighborhood of two other roots, $T_{1}=2.86046, T_{2}=2.98955$

\begin{tabular}{|c|c|c|}
\hline$T$ & $F(T)=T^{7}$ & $P_{3}(2, T)=-9028+13818 T-7140 T^{2}+1260 T^{3}$ \\
\hline 2.81000 & 1383.38875 & 1379.35766 \\
\hline 2.82000 & 1418.22050 & 1415.09168 \\
\hline 2.83000 & 1453.80129 & 1451.52962 \\
\hline 2.84000 & 1490.14449 & 1488.67904 \\
\hline 2.85000 & 1527.26367 & 1526.5475 \\
\hline 2.86046 & 1566.94022 & 1566.940217 \\
\hline 2.87000 & 1603.88519 & 1604.47178 \\
\hline 2.88000 & 1643.41563 & 1644.54272 \\
\hline 2.89000 & 1683.77827 & 1685.36294 \\
\hline 2.90000 & 1724.98763 & 1726.94 \\
\hline 2.91000 & 1767.05848 & 1769.28146 \\
\hline 2.92000 & 1810.00577 & 1812.39488 \\
\hline 2.93000 & 1853.84467 & 1856.28782 \\
\hline 2.94000 & 1898.59054 & 1900.96784 \\
\hline 2.95000 & 1944.25897 & 1946.4425 \\
\hline 2.96000 & 1990.86576 & 1992.71936 \\
\hline 2.97000 & 2038.42692 & 2039.80598 \\
\hline 2.98000 & 2086.95867 & 2087.70992 \\
\hline 2.98955 & $\underline{2134.25217}$ & $\underline{2134.252175}$ \\
\hline 3.00000 & 2187.00000 & 2186 \\
\hline 3.01000 & 2238.54314 & 2236.40126 \\
\hline 3.02000 & 2291.12403 & 2287.65008 \\
\hline 3.03000 & 2344.76002 & 2339.75402 \\
\hline 3.04000 & 2399.46868 & 2392.72064 \\
\hline $11.1 a b$ & &
\end{tabular}

Table 11. Table of values of $F(T)=T^{7}$ and $P_{3}(2, T)=-9028+13818 T-7140 T^{2}+1260 T^{3}$ in neighborhood of $T_{2}=2.8605, T_{3}=2.98955$.

Below we show graphically the intersection of $F(T)=T^{7}$ and $P_{3}(2, T)=-9028+13818 T-$ $7140 T^{2}+1260 T^{3}$ in point $T_{3}=3.98704$ in points $T_{2}=2.8605, T_{3}=2.98955$ 


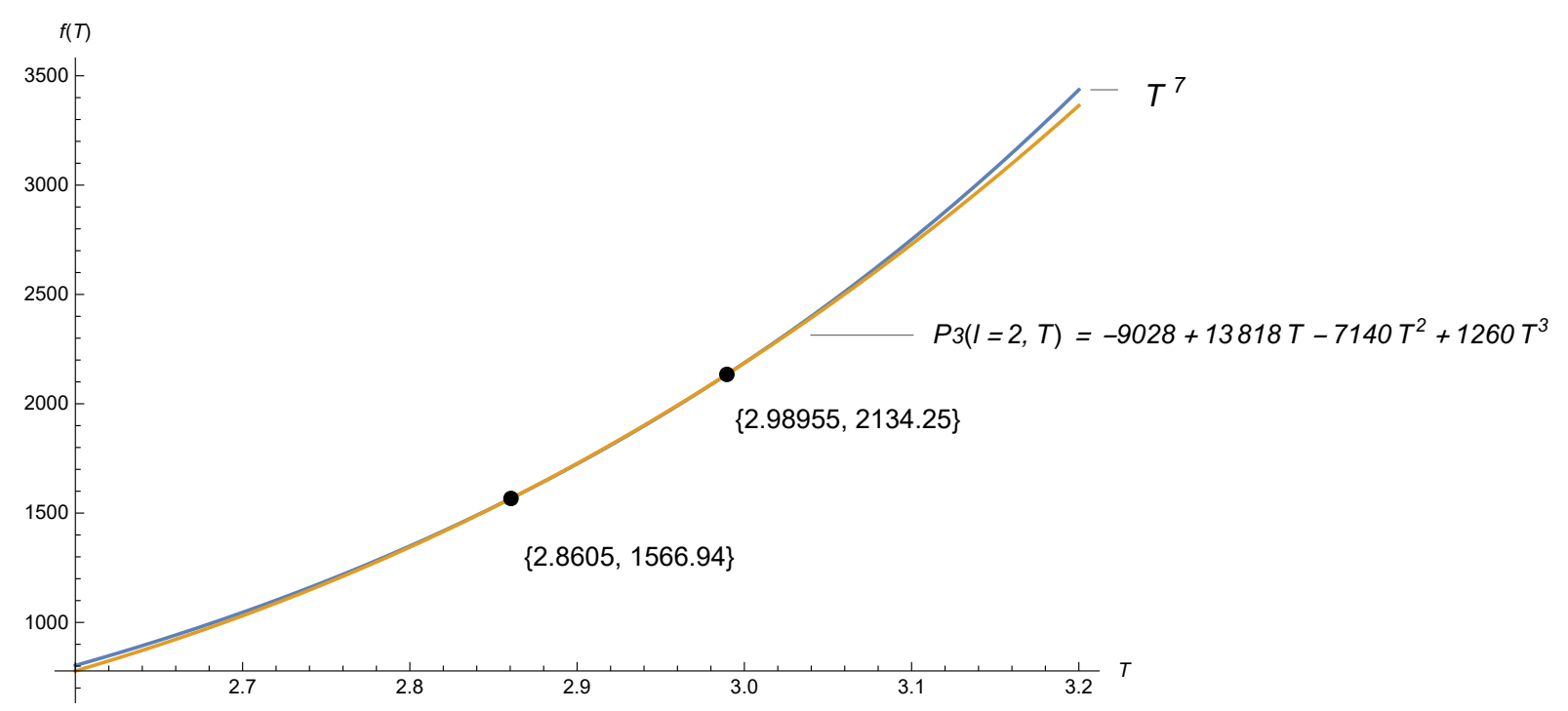

Figure 10. Intersection of $F(T)=T^{5}$ and $P_{2}(3, T)$ in points $T_{2}=2.8605, T_{3}=2.98955$.

\section{AcKnowledgements}

We would like to thank to Dr. Max Alekseyev (Department of Mathematics and Computational Biology, George Washington University) for sufficient help in the derivation of $A_{m, j}$ coefficients, Dr. Hansruedi Widmer for his useful comments concerning the derivation of coefficients $A_{m, j}$, Dr. Ron Knott (Visiting Fellow, Dept. of Mathematics at University of Surrey) for his useful suggestions on the writing of this article, and to Mr. Albert Tkaczyk his help in derivation of the sequences A300656, A300785. Also, we'd like to thank to OEIS editors Michel Marcus, Peter Luschny, Jon E. Schoenfield and others for their patient and faithful volunteer work and for useful comments and suggestions during the editing of sequences, concerned with this manuscript. We, also, greatly appreciate the preliminary proofread and suggestions by www.cantab.net/users/bnr/.

\section{Conclusion}

In this manuscript we have derived and discussed the polynomials $P_{m}(\ell, T)$, such that converge to the monomial $T^{2 m+1}, m \in \mathbb{N}_{0}$ as $T \rightarrow \ell, \ell \in \mathbb{N}$. Polynomial $P_{m}(\ell, T)$ gives weak approximation of the monomial $T^{2 m+1}$ mainly in neighborhood of the solution of the equation $T^{2 m+1}-P_{m}(\ell, T)=0$.

\section{Supplementary Files}

We provide the following supplementary files to support our study:

- https://kolosovpetro.github.io/arxiv_1603_02468/identity_1_1.txt- Mathematica program, implementation the l.h.s of identity $\left[1.1\right.$, that is $T^{2 m+1}=\sum_{k=1}^{\ell} \sum_{j=0}^{m} A_{m, j} k^{j}(T-k)^{j}, \ell=$ $T \in \mathbb{N}$.

- https://kolosovpetro.github.io/arxiv_1603_02468/identity_1_1_r_h_s.txt- Mathematica program, implementation the r.h.s of identity (1.1), that is $T^{2 m+1}=\sum_{k=0}^{m}(-1)^{m-k} U_{m}(\ell, k) \cdot T^{k}, \ell=$ $T \in \mathbb{N}$.

- https://kolosovpetro.github.io/arxiv_1603_02468/u_coefficients_in_row.txt - Mathematica program, lists the coefficients $U_{m}(\ell, k)$ in (1.1) for given $m$. (By default $m=2$ )

\section{REFERENCES}

[1] Donald E. Knuth., Johann Faulhaber and Sums of Powers, pp. 9-10., arXiv preprint, arXiv:math/9207222v1[math.CA], 1992.

[2] John Riordan, Combinatorial Identities (New York: John Wiley \& Sons, 1968).

[3] Petro, Kolosov, et al., Entry "Triangle read by rows: $T(n, k)=6 *(n-k) * k+1$ ", A287326, 2017.

[4] Weisstein, Eric W. "Finite Difference.' From Mathworld

[5] Joe Keane, et al., Entry "6 times triangular numbers: $a(n)=3 * n *(n+1)$ ", A028896, 1999.

[6] Joshua Giambalvo, et al., Entry " $a(n)=2 * n^{3}+3 * n^{2} "$, A275709, 2016. 
[7] Weisstein, Eric W. "Bernoulli Number.' From Mathworld-A Wolfram Web Resource.

[8] Petro, Kolosov, et al., Entry "Triangle read by rows: $T(n, k)=30 *(n-k)^{2} * k^{2}+1$ ", A300656, 2018.

[9] Petro, Kolosov, et al., Entry "Triangle read by rows: $T(n, k)=140 *(n-k)^{3} * k^{3} *-14 *(n-k) * k+1 ", A 300785$, 2018.

[10] Ronald Graham, Donald Knuth, and Oren Patashnik (1989-01-05). "Binomial coefficients". Concrete Mathematics (1st ed.). Addison Wesley Longman Publishing Co. p. 162. ISBN 0-201-14236-8, online copy.

[11] Johann Faulhaber, Academia Algebræ, Darinnen die miraculosische Inventiones zuden höchsten Cossen weiters continuirt und profitiert werden. Augspurg, bey Johann Ulrich Schönigs, 1631. (Call number QA154.8 F3 1631a f MATH at Stanford University Libraries.), online copy.

[12] Donald E. Knuth., Two notes on notation., pp. 1-2, arXiv preprint, arXiv:math/9205211 [math.HO], 1992.

[13] Petro, Kolosov, et al., Entry "Triangle read by rows: $\operatorname{Numeator}\left(A_{m, j}\right), 0 \leq j \leq m, m \geq 0 ", A 302971,2018$.

[14] Petro, Kolosov, et al., Entry "Triangle read by rows: Denominator $\left(A_{m, j}\right), 0 \leq j \leq m, m \geq 0$ ", A304042, 2018.

[15] N. J. A. Sloane, et al., Entry " $a(n)=(2 n+1) ! / n !^{2} "$, A002457.

[16] Donald E. Knuth, The Art of Computer Programming Vol. 3, (1973) Addison-Wesley, Reading, MA.

[17] Petro, Kolosov, et al., Entry "Consider coefficients $U_{m}(n, k)$ defined by the identity $\sum_{k=1 . . l} \sum_{j=0 . . m} A 302971(m, j) / A 304042(m, j) * k^{j} *(t-k)^{j}=\sum_{k=0 . . m}(-1)^{(m-k)} * U_{m}(l, k) * t^{k}$ that holds for all positive integers $l, m, t$. This sequence gives 3 -column table read by rows, where $n$-th row lists coefficients $U_{2}(n, k)$ for $k=0,1,2 . "$, A316349, 2018.

[18] Petro, Kolosov, et al., Entry "Consider coefficients $U_{m}(n, k)$ defined by the identity $\sum_{k=1 . . l} \sum_{j=0 . . m} A 302971(m, j) / A 304042(m, j) * k^{j} *(t-k)^{j}=\sum_{k=0 . . m}(-1)^{(m-k)} * U_{m}(l, k) * t^{k}$ that holds for all positive integers $l, m, t$. This sequence gives 4 -column table read by rows, where $n$-th row lists coefficients $U_{3}(n, k)$ for $k=0,1,2,3 . "$, A316387, 2018.

[19] John Riordan, Combinatorial Identities (New York: John Wiley \& Sons, 1968).

[20] Petro Kolosov, Peter Luschny, Entry "Triangle read by rows: coefficients in the sum of odd powers as expressed by Faulhaber's theorem, $T(n, k)$ for $n \geq 1,1 \leq k \leq n$ ", A303675 in [9], 2018.

E-mail address: kolosovp94@gmail.com

$U R L$ : https://kolosovpetro.github.io 